\title{
Pittsburgh air quality study overview
}

\author{
Ann E. Wittig ${ }^{\mathrm{a}, *}$, Natalie Anderson ${ }^{\mathrm{b}}$, Andrei Y. Khlystov ${ }^{\mathrm{c}}$, Spyros N. Pandis ${ }^{\mathrm{d}, \mathrm{e}}$, \\ Cliff Davidson $^{\mathrm{b}, \mathrm{e}}$, Allen L. Robinson ${ }^{\mathrm{e}, \mathrm{f}}$ \\ ${ }^{a}$ Department of Civil Engineering, City College of New York, New York, NY 10031, USA \\ ${ }^{\mathrm{b}}$ Department of Civil and Environmental Engineering, Carnegie Mellon University, Pittsburgh, PA 15213, USA \\ ${ }^{\mathrm{c}}$ Department of Civil and Environmental Engineering, Duke University, Durham, NC 27708, USA \\ ${ }^{\mathrm{d}}$ Department of Engineering and Public Policy, Carnegie Mellon University, Pittsburgh, PA 15213, USA \\ ${ }^{\mathrm{e}}$ Department of Chemical Engineering, Carnegie Mellon University, Pittsburgh, PA 15213, USA \\ ${ }^{\mathrm{f}}$ Department of Mechanical Engineering, Carnegie Mellon University, Pittsburgh, PA 15213, USA
}

\begin{abstract}
Ambient sampling for the Pittsburgh Air Quality Study (PAQS) was conducted from July 2001 to September 2002. The study was designed (1) to characterize particulate matter (PM) by examination of size, surface area, and volume distribution, chemical composition as a function of size and on a single particle basis, morphology, and temporal and spatial variability in the Pittsburgh region; (2) to quantify the impact of the various sources (transportation, power plants, biogenic sources, etc.) on the aerosol concentrations in the area; and (3) to develop and evaluate the next generation of atmospheric aerosol monitoring and modeling techniques. The PAQS objectives, study design, site descriptions and routine and intensive measurements are presented. Special study days are highlighted, including those associated with elevated concentrations of daily average $\mathrm{PM}_{2.5}$ mass. Monthly average and diurnal patterns in aerosol number concentration, and aerosol nitrate, sulfate, elemental carbon, and organic carbon concentrations, light scattering as well as gas-phase ozone, nitrogen oxides, and carbon monoxide are discussed with emphasis on the processes affecting them. Preliminary findings reveal day-to-day variability in aerosol mass and composition, but consistencies in seasonal average diurnal profiles and concentrations. For example, the seasonal average variations in the diurnal $\mathrm{PM}_{2.5}$ mass were predominately driven by the sulfate component.
\end{abstract}

(C) 2004 Elsevier Ltd. All rights reserved.

Keywords: Atmospheric aerosol; Supersite; PAQS

\section{Introduction}

Airborne particulate matter (PM) continues to pose serious health risks for susceptible members of the population and for sensitive ecosystems. The design of cost effective PM control strategies is limited by the lack of understanding of the relationship between PM and health effects. This lack of understanding is exacerbated by a paucity of physiological data, the difficulty of establishing the PM source-receptor relationships, and finally the limitations of existing instrumentation for PM measurements. The Pittsburgh Air Quality Study (PAQS) is a comprehensive multidisciplinary set of

\footnotetext{
*Corresponding author.
}

projects in the Pittsburgh region, designed to address the above issues. PAQS was led by Carnegie Mellon University (CMU) and included contributions by investigators from fifteen universities, a national laboratory, three private companies, and state and local air pollution agencies, as noted in Table 1. PAQS was supported by the Environmental Protection Agency (EPA) Supersites Program and the Department of Energy National Energy Technology Laboratory (NETL).

In this paper, we present the overall study design and features of the PAQS dataset. We begin by summarizing the PAQS program objectives and hypotheses, and describing the study area and measurement sites. We then describe the measurement methods and the 
Table 1

PAQS contributors

\begin{tabular}{ll}
\hline Contribution & Affiliation \\
\hline Overall project management, Aerosol physical and chemical characterization & Carnegie Mellon Univ.
\end{tabular}

Field manager

Quality assurance manager

Aircraft measurements

Satellite site measurements

Satellite site measurements

Satellite site measurements

Satellite site measurements

Additional instrumentation

Single particle morphology

Single particle mass spectroscopy

Semi-continuous size resolved aerosol composition

Semi-continuous metal measurements

Semi-continuous nitrate and sulfate measurements

Semi-continuous OC/EC measurements, FTIR spectroscopy of aerosols

Biaerosol composition

Integrated organic aerosol measurements

Integrated organic aerosol speciation

PM surface area concentration measurements

Semi-continuous VOC measurements

Continuous peroxide measurements, cloud and fog composition

Source-receptor relationships

Meteorology

Univ. of Maryland
ACHD
NETL
Ohio Univ.
PA DEP
CONSOL

RJ Lee Group, Ltd.

Univ. of California at Davis, Univ. of Delaware

Univ. of Colorado, AERODYNE

Univ. of Maryland

Aerosol Dynamics, Inc.

Rutgers Univ.

Univ. of Colorado

Brigham Young Univ.

Florida Intern. Univ.

Paul Scherrer Institute

Univ. of California at Berkeley

Colorado State Univ.

Clarkson Univ.

Univ. of Wisconsin
Contributor

Cliff Davidson, Spyros Pandis, Allen Robinson Andrey Khlystov Ann (Beth) Wittig

Bruce Doddridge

Darrell Stern

Richard Anderson

Kevin Crist

Various individuals

Steve Winter

Gary Casuccio

Anthony Wexler,

Murray Johnson

Jose Jimenez,

Doug Worsnop

John Ondov

Susanne Hering

Barbara Turpin

Mark Hernandez

Delbert Eatough

Wolfgang Rogge

Urs Baltensperger

Allen Goldstein

Jeff Collett

Phil Hopke

Jonathan Kahl availability of the measurements for both routine monitoring and intensive study periods. Next, we highlight the general features of the measurements collected at the central site, by examining monthly average and diurnal meteorological parameters and ambient concentrations of gases, aerosols, and aerosol species.

\section{Program objectives}

PAQS was designed to achieve several objectives: to determine the physical and chemical characteristics of PM in the Pittsburgh region; to develop and evaluate the next generation of atmospheric aerosol monitoring techniques; to update emission profiles for important regional sources; to quantify the impact of the various sources on the local PM concentrations; and to predict changes in the PM characteristics due to proposed changes in emissions. The last objective was based on concurrent modeling studies and was designed to support the development of regulations. These objectives were addressed through four components of the research: (1) ambient monitoring at a central site and a set of satellite sites in the region; (2) an instrument development and evaluation study; (3) a data analysis and synthesis component; and (4) a comprehensive modeling component. 


\section{Hypotheses and their testing}

Approximately 20 hypotheses were proposed to meet the goals of PAQS. These hypotheses address ambient aerosol measurement issues, questions regarding atmospheric processes and aerosol properties, and the need for refinement of source-receptor relationships. The key hypotheses are summarized in Table 2. A complete list of hypotheses and a description of the hypotheses testing can be found in the PAQS Quality Assurance Project Plan (Khlystov et al., 2001).

Table 2

Primary hypotheses to be investigated during PAQS

\section{Ambient aerosol characterization}

1.1: The measured aerosol mass can be fully explained if the water retained by organics and inorganics, the full organic aerosol contribution, and the full crustal contribution is accounted for.

1.2: The ambient aerosol surface area can be calculated with an error of less than $20 \%$ by using aerodynamic size measurements and by assuming that all particles are spherical.

\section{Measurement methods}

2.1: Single particle mass spectrometers can be used to obtain the full number and mass composition distributions of ambient aerosols.

2.2: Semi-continuous nitrate, sulfate, carbon and elements measurement techniques can quantify concentrations under conditions prevalent at the site.

\section{PAQS supersite description}

There are roughly two million people living in the Pittsburgh Metropolitan Statistical Area, and elderly people represent a significant fraction of the population. The area is located between the utilities and agricultural sources of the Midwest and the large urban centers of the East (Fig. 1). The central site was located on a grassy hill in Schenley Park, approximately $6 \mathrm{~km}$ east of downtown Pittsburgh. It was separated from the city in the predominant upwind direction (south and west) by roughly $1 \mathrm{~km}$ of parkland. It was at least several hundred meters from any other major

\section{Source-receptor relationships}

4.1: A complementary suite of instruments and techniques can be used to directly determine the local air quality contributions from a broad range of primary and secondary sources.

4.2: An increase in temporal resolution of elemental constituents of atmospheric aerosol coupled with sulfate and carbon analyses of comparable frequencies will permit unprecedented resolution of sources by receptor modeling techniques.

4.3: Specific aerosol signatures are associated with transport from specific source regions and along different altitudes.

\section{Aerosol properties}

5.1: Visibility in the area can be predicted from $\mathrm{RH}$ and size/ composition information obtained from aerosol sizing instruments and size bulk chemistry measurements.

5.2: Most particles in the area are liquid throughout the day in both winter and summer.

5.3: Aerosol in the area consists of two groups of particles based on the hygroscopic properties: those consisting mainly of sulfates that grow rapidly with relative humidity and those consisting of mainly carbonaceous material that grow slowly.

\section{Atmospheric processes}

3.1: Aerosol nucleation can be a major source of aerosol number in both urban and rural areas in the study region. 3.2: Biogenic primary and secondary aerosols are a major component of the organic aerosol in the Pittsburgh region. 3.3: Fogs and low clouds are responsible for extreme acid sulfate conditions in the Pittsburgh region.

3.4: The response of $\mathrm{PM}_{2.5}$ to changes in sulfate is highly nonlinear during the winter, is linear during the summer, and is controlled by the ammonia availability.

3.5: The secondary aerosol contribution to organic carbon (OC) exceeds $50 \%$ during the peak PM concentration days, but is approximately $20 \%$ on a yearly average basis.

3.6: The regional contributions to the $\mathrm{PM}_{2.5}$ levels in the Pittsburgh region for some compounds exceed the local contribution, whereas for others the local contributions exceed the regional contributions 
source of air pollution: the site was positioned approximately $50 \mathrm{~m}$ past the end of a dead end street, and several hundred meters from the nearest heavily traveled street (Forbes Avenue). The position of the central site in relation to the downtown Pittsburgh area is shown in Fig. 2. Sampling equipment was housed in an air pollution monitoring station, with samplers and instrument inlets on the station rooftop (Fig. 2).

Five additional sites were used for satellite measurements (Fig. 1). The Florence site was about $50 \mathrm{~km}$ west of the main site in a rural area with no nearby sources. The Greensburg site was about $50 \mathrm{~km}$ east of the main site in a suburban area, close to a heavily traveled road but otherwise in an area of only moderate traffic and few

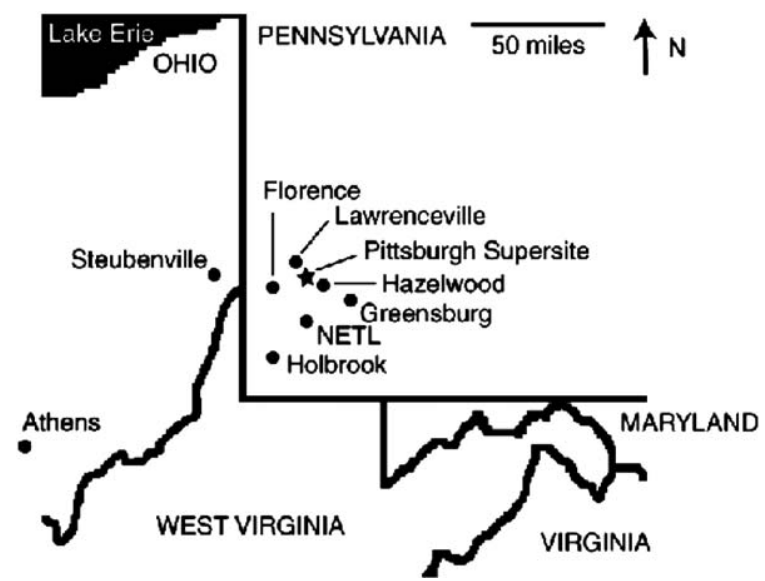

Fig. 1. Map of the PAQS region showing the location of the central site, the Florence, Lawrenceville, Hazelwood, Greensburg, and Athens satellite sites, and the NETL, Holbrook, and Steubenville sites independently operated by other researchers during PAQS. stationary sources. Satellite sites were also located in the Lawrenceville and Hazelwood neighborhoods of the City of Pittsburgh. The final satellite site was located in Athens, $\mathrm{OH}$. Additional air quality measurements were conducted concurrently by others at the NETL laboratories, approximately $15 \mathrm{~km}$ south of downtown Pittsburgh, at Holbrook, PA and at Steubenville, $\mathrm{OH}$ (Fig. 1). Table 3 lists the latitude, longitude, and period of operation of these sites. In addition to the groundbased measurements, two aircraft provided air quality and meteorological data aloft during the intensive periods. R. B. Jacko and Associates, Inc. operated a Cessna 210 Turbo Centurion and the University of Maryland operated a Piper 23-250 Aztec-F (Table 4).

\section{PAQS measurements}

Baseline monitoring took place over a 14-month period that began 1 July, 2001, and provided data of a wide variety of PM physical and chemical characteristics, including biological aerosol composition; gaseous species and fogwater composition; and meteorological parameters. Intensive monitoring was performed during two periods, from 1 July to 3 August, 2001 (ESP01) and 1 January to 15 January, 2002 (ESP02). During these periods, the temporal resolution of these baseline measurements was increased and the baseline measurements were supplemented with measurements of particle morphology, size resolved aerosol composition, organic aerosol speciation, semi-continuous aerosol metal composition and gaseous volatile organic compound (VOC) speciation. The frequency, resolution, and dates over which each method was used at the central site are summarized in Table 5. Quality control procedures used
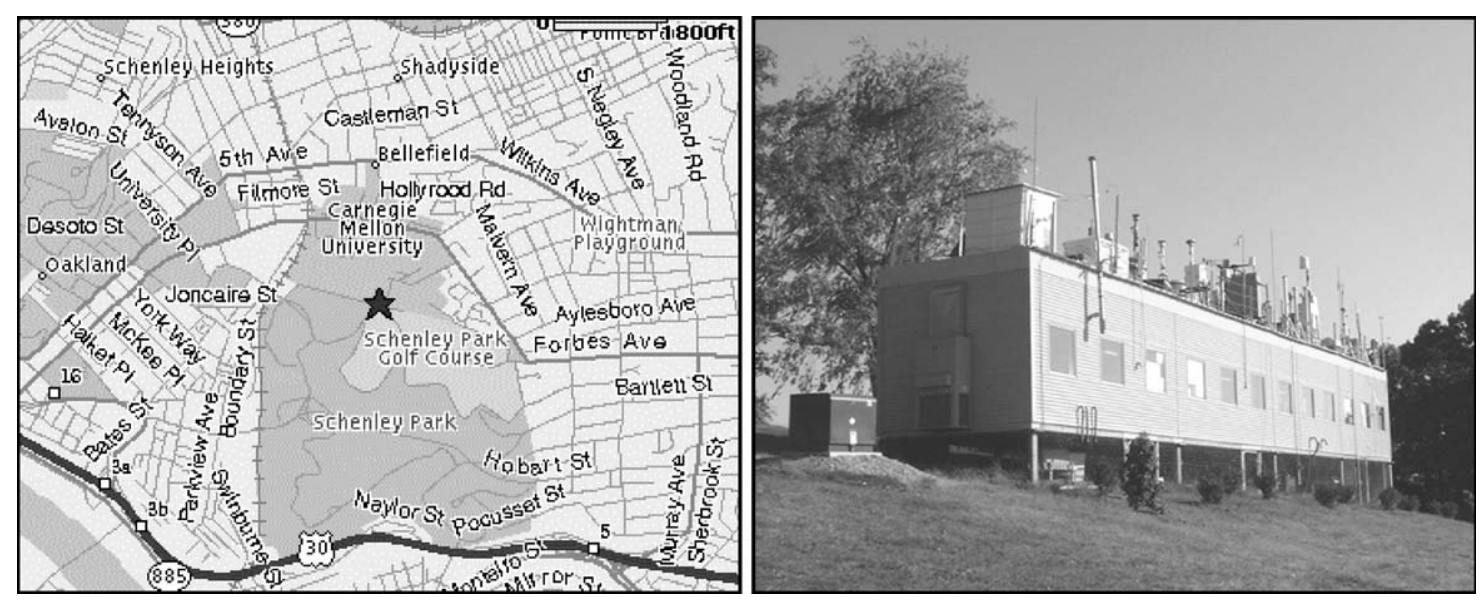

Fig. 2. Map of the downtown Pittsburgh area showing the approximate location of the central site (indicated by a star) and a photograph of the site from the northwest. 
Table 3

Locations of measurements collected during PAQS

\begin{tabular}{llll}
\hline Site name & Group $^{\text {a }}$ & Latitude/Longitude (decimal degrees) & Period \\
\hline Central site $^{\mathrm{b}}$ & CMU & $40.4395 /-79.9405$ & $6 / 30 / 01-7 / 31 / 02$ \\
Satellite sites & & & \\
Hazelwood, PA & & $40.4124 /-79.9424$ & $6 / 30-7 / 30 / 01,1 / 2-1 / 22 / 02$ \\
Lawrenceville, PA & CMU/ACHD & $40.4432 /-79.9595$ & $6 / 30-7 / 30 / 01,1 / 2-1 / 22 / 02$ \\
Florence, PA & CMU/ACHD & $40.4454 /-80.4212$ & $6 / 30-7 / 30 / 01,1 / 2-1 / 22 / 02$ \\
& & & $1 / 3-1 / 22 / 02,7 / 14-8 / 3 / 02^{\mathrm{d}}$ \\
Greensburg, PA & CMU/DEP & $40.3043 /-79.5059$ & $2 / 24 / 02$ to $3 / 28 / 02^{\mathrm{e}}$ \\
Athens, OH & Ohio Univ. & $39.3283 /-82.9067$ & $6 / 30-7 / 30 / 01,1 / 2-1 / 22 / 02$ \\
& & & $6 / 30-8 / 8 / 01$ \\
Aircraft & & & $7 / 6-8 / 2 / 01$ \\
Cessna & & Various & $7 / 6-8 / 1 / 01,7 / 2-8 / 4 / 02$ \\
Piper & Jacko & Various & $6 / 30-8 / 8 / 01$ \\
Non-PAQS sites & UM & & $6 / 30-8 / 8 / 01$ \\
NETL & & $40.3065 /-79.9794$ & $6 / 30-8 / 8 / 01$ \\
Holbrook, PA & NETL & $39.8160 /-80.2850$ & \\
Steubenville, OH & ATS & $40.3617 /-80.6147$ & \\
\hline
\end{tabular}

${ }^{a}$ Groups-ACHD: Allegheny County Health Department; ATS: Advanced Technology Systems; DEP: Pennsylvania Department of Environmental Planning; NETL: National Energy and Technology Laboratory; UM: University of Maryland; Jacko: R.B. Jacko and Associates.

${ }^{\mathrm{b}}$ Central site measurements - see Table 5 .

${ }^{\mathrm{c}}$ Satellite site measurements- $\mathrm{PM}_{2.5}$ mass, $\mathrm{PM}_{2.5}$ elements, and $\mathrm{PM}_{2.5}$ ammonium, nitrate, potassium, sodium, sulfate, elemental carbon, and organic carbon.

${ }^{\mathrm{d}}$ The CMU denuded organic sampler was used to measure $24 \mathrm{~h}$ organic carbon and elemental carbon concentrations on 17 days within the stated range.

${ }^{\mathrm{e}}$ Particle sizing instruments (TSI 3071 DMA and $3010 \mathrm{CPC}$ ) were used to measure $10 \mathrm{~min}$ aerosol size distribution semi-continuously from 0.012 to $0.280 \mu \mathrm{m}$.

${ }^{\mathrm{f}}$ Aircraft measurements-see Table 4.

Table 4

Summary of the aircraft flights completed during PAQS

\begin{tabular}{llll}
\hline Date of flight & Area of operation & Group & Flight details \\
\hline $7 / 6 / 2001 \mathrm{a}$ & Pittsburgh, PA & UM $^{\mathrm{a}}$ & Cadiz, OH to Sebring, OH; Sebring, OH to Cadiz, OH. \\
$7 / 6 / 2001 \mathrm{~b}$ & Western PA, IN & Jacko $^{\mathrm{b}}$ & Allegheny, PA to Pittsburgh, PA to Lafayette, IN \\
$7 / 22 / 2001$ & Pittsburgh, PA & SM & Steubenville, OH to Tarentum, PA to Monongahela, PA \\
$7 / 23 / 2001 \mathrm{a}$ & Pittsburgh, PA & UM & Steubenville, OH to Tarentum, PA to Monongahela, PA \\
$7 / 23 / 2001 \mathrm{~b}$ & Pittsburgh, PA & UM & Steubenville, OH to Tarentum, PA to Monongahela, PA \\
$7 / 23 / 2001 \mathrm{c}$ & Pittsburgh, PA & Jacko & Lafayette, IN to Pittsburgh, PA to Lafayette, IN \\
$8 / 1 / 2001 \mathrm{a}$ & Pittsburgh, PA & UM & Steubenville, OH \\
$8 / 1 / 2001 \mathrm{~b}$ & Pittsburgh, PA & UM & Monongahela, PA to Tarentum, PA \\
$8 / 2 / 2001 \mathrm{a}$ & IN, Western PA & Jacko & Lafayette, IN to Pittsburgh, PA \\
$8 / 2 / 2001 \mathrm{~b}$ & OH, Western PA & Jacko & Pittsburgh, PA to Columbus, OH \\
$7 / 2 / 2002 \mathrm{a}$ & MD, Western PA & UM & Fort Meade, MD to Bedford, PA \\
$7 / 2 / 2002 \mathrm{~b}$ & Western PA & UM & Clarion, PA to Corry, PA \\
$8 / 4 / 2002 \mathrm{a}$ & Western PA & UM & Fort Meade, MD to Indiana, PA \\
8/4/2002b & Western PA, Central NY & UM & Wellsboro, PA to Cortland, NY \\
\hline
\end{tabular}

${ }^{\mathrm{a}} \mathrm{UM}$ (University of Maryland) measurements-Ozone (4 s resolution); NO (10 s resolution); $\mathrm{NO}_{2}$ (undetermined resolution); $\mathrm{CO}$ and $\mathrm{SO}_{2}$ (1 min resolution); aerosol absorption and scattering (1 min resolution); temperature, relative humidity, pressure, latitude, longitude, elevation (10 s resolution).

${ }^{\mathrm{b}}$ Jacko (R. B. Jacko and Associates, Inc.) measurements-Ozone (4 s resolution); $\mathrm{NO}_{x}$ ( $10 \mathrm{~s}$ resolution); VOC and Carbonyls (15 min or longer samples); aerosol scattering (1 min resolution); $\mathrm{PM}_{2.5}$ and $\mathrm{SO}_{2}, \mathrm{NH}_{3}$, and $\mathrm{HNO}_{3}$ (2 $\mathrm{h}$ or longer samples); temperature, relative humidity, pressure, ultraviolet radiation, latitude, longitude, elevation (10 s resolution). 
Table 5

Summary of PAQS measurements

\begin{tabular}{|c|c|c|c|c|c|}
\hline Observable & Method $^{\mathrm{a}}$ & Group $^{b}$ & Resolution & Frequency & Period of operation $^{c}$ \\
\hline \multicolumn{6}{|l|}{ Aerosol mass } \\
\hline $\mathrm{PM}_{10}$ mass & $\begin{array}{l}\text { Dichotomous sampler with } \\
\text { gravimetry }\end{array}$ & $\mathrm{CMU}$ & $24 \mathrm{~h}$ & Daily & $7 / 1 / 01-7 / 1 / 02$ \\
\hline $\mathrm{PM}_{2.5}$ mass & $\begin{array}{l}\text { Dichotomous sampler with } \\
\text { gravimetry }\end{array}$ & $\mathrm{CMU}$ & $24 \mathrm{~h}$ & Daily & $7 / 1 / 01-7 / 1 / 02$ \\
\hline $\mathrm{PM}_{2.5}$ mass & $\begin{array}{l}\text { Federal Reference Method } \\
\text { sampler with gravimetry }\end{array}$ & CMU & $24 \mathrm{~h}$ & Daily & $7 / 1 / 01-7 / 1 / 02$ \\
\hline $\mathrm{PM}_{2.5}$ mass & $\begin{array}{l}\text { R\&P 1400A TEOM monitor } \\
\text { with SES }\end{array}$ & $\mathrm{CMU}$ & $10 \mathrm{~min}$ & Continuous & $7 / 1 / 01-9 / 1 / 02$ \\
\hline $\mathrm{PM}_{x}$ mass & $\begin{array}{l}\text { MOUDI sampler with } \\
\text { gravimetry }\end{array}$ & $\mathrm{CMU}$ & $24 \mathrm{~h}$ & Daily & $7 / 1 / 01-7 / 1 / 02^{\mathrm{d}}$ \\
\hline $\mathrm{PM}_{x}$ mass & $\begin{array}{l}\text { MOUDI sampler with } \\
\text { gravimetry }\end{array}$ & $\mathrm{CMU}$ & $8 \mathrm{~h}$ & 3 per day & $7 / 22-25 / 01,7 / 31-8 / 3 / 01$ \\
\hline \multicolumn{6}{|l|}{$\begin{array}{l}\text { Aerosol size } \\
\text { distribution }\end{array}$} \\
\hline $\begin{array}{l}\text { Aerosol number } \\
\text { distribution }\end{array}$ & $\begin{array}{l}\text { TSI ultra fine SMPS, SMPS } \\
\text { and APS }\end{array}$ & CMU & $10 \mathrm{~min}$ & Continuous & $7 / 1 / 01-7 / 1 / 02$ \\
\hline $\begin{array}{l}\text { Aerosol surface area } \\
\text { distribution }\end{array}$ & $\begin{array}{l}\text { TSI ultra fine SMPS, SMPS } \\
\text { and APS }\end{array}$ & $\mathrm{CMU}$ & $10 \mathrm{~min}$ & Continuous & $7 / 1 / 01-7 / 1 / 02$ \\
\hline $\begin{array}{l}\text { Aerosol surface area } \\
\text { distribution }\end{array}$ & Epiphaniometer & PSI & $30 \mathrm{~min}$ & Continuous & $6 / 11 / 01-9 / 18 / 01$ \\
\hline $\begin{array}{l}\text { Aerosol volume } \\
\text { distribution }\end{array}$ & $\begin{array}{l}\text { TSI ultra fine SMPS, SMPS } \\
\text { and APS }\end{array}$ & CMU & $10 \mathrm{~min}$ & Continuous & $7 / 1 / 01-7 / 1 / 02$ \\
\hline \multicolumn{6}{|l|}{$\begin{array}{l}\text { Aerosol chemical } \\
\text { composition }\end{array}$} \\
\hline $\mathrm{PM}_{2.5}$ inorganic ions & CMU sampler with IC analysis & $\mathrm{CMU}$ & $24 \mathrm{~h}$ & Daily & $7 / 1 / 01-7 / 1 / 02^{\mathrm{d}}$ \\
\hline $\mathrm{PM}_{2.5}$ inorganic ions & CMU sampler with IC analysis & CMU & $4-6 h$ & 5 per day & ESP01 Intensive \\
\hline $\mathrm{PM}_{x}$ inorganic ions & $\begin{array}{l}\text { MOUDI sampler with IC } \\
\text { analysis }\end{array}$ & $\mathrm{CMU}$ & $24 \mathrm{~h}$ & Daily & $7 / 1 / 01-7 / 1 / 02^{\mathrm{d}}$ \\
\hline $\mathrm{PM}_{x}$ inorganic ions & $\begin{array}{l}\text { MOUDI sampler with IC } \\
\text { analysis }\end{array}$ & CMU & $8 \mathrm{~h}$ & 3 per day & $7 / 22-25 / 01,7 / 31-8 / 3 / 01$ \\
\hline $\mathrm{PM}_{10}$ elements & $\begin{array}{l}\text { Hi-Vol sampler with ICP-MS } \\
\text { analysis }\end{array}$ & CMU & $24 \mathrm{~h}$ & Daily & $7 / 12 / 01-8 / 02 / 02$ \\
\hline $\mathrm{PM}_{2.5}$ elements & $\begin{array}{l}\text { Hi-Vol sampler with ICP-MS } \\
\text { analysis }\end{array}$ & $\mathrm{CMU}$ & $24 \mathrm{~h}$ & Daily & $7 / 11 / 01-9 / 30 / 02$ \\
\hline $\mathrm{PM}_{x}$ elements & $\begin{array}{l}\text { MOUDI sampler with ICP-MS } \\
\text { analysis }\end{array}$ & $\mathrm{CMU}$ & $24 \mathrm{~h}$ & Daily & $\begin{array}{l}\text { ESP01, ESP02 } \\
\text { Intensives }^{\mathrm{d}}\end{array}$ \\
\hline $\mathrm{PM}_{x}$ elements & $\begin{array}{l}\text { MOUDI sampler with ICP-MS } \\
\text { analysis }\end{array}$ & CMU & $8 \mathrm{~h}$ & 3 per day & $7 / 22-25 / 01,7 / 31-8 / 3 / 01$ \\
\hline $\begin{array}{l}\mathrm{PM}_{2.5} \text { organic and } \\
\text { elemental carbon }\end{array}$ & $\begin{array}{l}\text { CMU TQQQ sampler with } \\
\text { TOT analysis }\end{array}$ & $\mathrm{CMU}$ & $24 \mathrm{~h}$ & Daily & $7 / 1 / 01-7 / 31 / 02^{\mathrm{d}}$ \\
\hline $\begin{array}{l}\mathrm{PM}_{2.5} \text { organic and } \\
\text { elemental carbon }\end{array}$ & $\begin{array}{l}\text { CMU TQQQ sampler with } \\
\text { TOT analysis }\end{array}$ & $\mathrm{CMU}$ & $4-6 h$ & 5 per day & ESP01 Intensive \\
\hline $\begin{array}{l}\mathrm{PM}_{2.5} \text { organic and } \\
\text { elemental carbon }\end{array}$ & $\begin{array}{l}\text { CMU denuded organic sampler } \\
\text { with TOT analysis }\end{array}$ & CMU & $24 \mathrm{~h}$ & 6th day & $7 / 1 / 01-6 / 1 / 02^{\mathrm{d}}$ \\
\hline $\begin{array}{l}\mathrm{PM}_{2.5} \text { organic and } \\
\text { elemental carbon }\end{array}$ & $\begin{array}{l}\text { CMU denuded organic sampler } \\
\text { with TOT analysis }\end{array}$ & $\mathrm{CMU}$ & $24 \mathrm{~h}$ & Daily & ESP01, ESP02 Intensives \\
\hline $\begin{array}{l}\mathrm{PM}_{2.5} \text { organic and } \\
\text { elemental carbon }\end{array}$ & $\begin{array}{l}\text { MOUDI sampler with TOT } \\
\text { analysis }\end{array}$ & $\mathrm{CMU}$ & $24 \mathrm{~h}$ & Daily & ESP01 Intensive $^{\mathrm{d}}$ \\
\hline $\begin{array}{l}\mathrm{PM}_{x} \text { organic and } \\
\text { elemental carbon }\end{array}$ & $\begin{array}{l}\text { MOUDI sampler with TOT } \\
\text { analysis }\end{array}$ & $\mathrm{CMU}$ & $8 \mathrm{~h}$ & 3 per day & $7 / 22-25 / 01,7 / 31-8 / 3 / 01$ \\
\hline $\begin{array}{l}\mathrm{PM}_{x} \text { organic and } \\
\text { elemental carbon }\end{array}$ & BYU PC-BOSS & BYU & $24 \mathrm{~h}$ & Daily & $7 / 9 / 01-7 / 31 / 01$ \\
\hline $\mathrm{PM}_{2.5}$ speciated & LPI sampler with FTIR & CMU & $24 \mathrm{~h}$ & Daily & ESP01, ESP02 Intensives \\
\hline
\end{tabular}


Table 5 (continued)

\begin{tabular}{|c|c|c|c|c|c|}
\hline Observable & Method $^{\mathrm{a}}$ & Group $^{\mathrm{b}}$ & Resolution & Frequency & Period of operation $^{c}$ \\
\hline $\begin{array}{l}\mathrm{PM}_{2.5} \text { speciated } \\
\text { organics }\end{array}$ & $\begin{array}{l}\text { CMU organic speciation } \\
\text { sampler }\end{array}$ & FIU & $24 \mathrm{~h}$ & 6th Day & $7 / 1 / 01-7 / 1 / 02^{\mathrm{d}}$ \\
\hline $\begin{array}{l}\mathrm{PM}_{2.5} \text { speciated } \\
\text { organics }\end{array}$ & $\begin{array}{l}\text { CMU organic speciation } \\
\text { sampler }\end{array}$ & FIU & $24 \mathrm{~h}$ & Daily & ESP01, ESP02 Intensives \\
\hline $\begin{array}{l}\mathrm{PM}_{2.5} \text { biological } \\
\text { material }\end{array}$ & $\begin{array}{l}\text { Epi-fluorescent microscopy } \\
\text { with assays }\end{array}$ & $\mathrm{UC}$ & $24 \mathrm{~h}$ & Daily & $7 / 7 / 01-7 / 1 / 02$ \\
\hline $\mathrm{PM}_{2.5}$ nitrate & $\begin{array}{l}\mathrm{R} \& \mathrm{P} 8400 \mathrm{~N} \text { with flash } \\
\text { volatilization }\end{array}$ & ADI & $10 \mathrm{~min}$ & $\begin{array}{l}\text { Semi- } \\
\text { continuous }\end{array}$ & $7 / 1 / 01-8 / 1 / 02$ \\
\hline $\mathrm{PM}_{2.5}$ sulfate & $\begin{array}{l}\text { R\&P } 8400 \text { S with flash } \\
\text { volatilization }\end{array}$ & ADI & $10 \mathrm{~min}$ & $\begin{array}{l}\text { Semi- } \\
\text { continuous }\end{array}$ & $7 / 1 / 01-9 / 1 / 02$ \\
\hline $\mathrm{PM}_{2.5}$ carbon & $\begin{array}{l}\text { ADI monitor with flash } \\
\text { volatilization }\end{array}$ & ADI & $30 \mathrm{~min}$ & $\begin{array}{l}\text { Semi- } \\
\text { continuous }\end{array}$ & $1 / 1 / 02-9 / 1 / 02$ \\
\hline $\mathrm{PM}_{2.5}$ carbon & $\begin{array}{l}\text { Denuded Sunset Labs in situ } \\
\text { TOT analyzer }\end{array}$ & RU & $2-4 h$ & $\begin{array}{l}\text { Semi- } \\
\text { continuous }\end{array}$ & $7 / 1 / 01-9 / 1 / 02$ \\
\hline $\begin{array}{l}\mathrm{PM}_{2.5} \text { water soluble } \\
\text { ions }\end{array}$ & $\begin{array}{l}\text { Khylstov steam sampler with } \\
\text { IC analysis }\end{array}$ & CMU & $1-2 \mathrm{~h}$ & $\begin{array}{l}\text { Semi- } \\
\text { continuous }\end{array}$ & $7 / 1 / 01-9 / 21 / 02$ \\
\hline $\begin{array}{l}\mathrm{PM}_{2.5} \text { water soluble } \\
\text { ammonium }\end{array}$ & Ammonium online detector & CMU & $10 \mathrm{~min}$ & Continuous & $7 / 1 / 01-9 / 21 / 02$ \\
\hline $\mathrm{PM}_{1.3}$ metals & SEAS with GFAA analysis & UMD & $30 \mathrm{~min}$ & $\begin{array}{l}\text { Semi- } \\
\text { continuous }\end{array}$ & $\begin{array}{l}7 / 8 /-8 / 10 / 01,3 / 29-4 / 17 / \\
02\end{array}$ \\
\hline $\begin{array}{l}\mathrm{PM}_{1.0} \text { size resolved } \\
\text { composition }\end{array}$ & $\begin{array}{l}\text { Aerodyne Mass Spectrometer } \\
\text { (AMS) }\end{array}$ & $\mathrm{UC}$, & & & AERODYNE \\
\hline $5 \mathrm{~min}$ & Semi-continuous & $\begin{array}{l}9 / 6 / 02-9 / 21 / \\
02\end{array}$ & & & \\
\hline \multicolumn{6}{|l|}{ Aerosol characteristics } \\
\hline $\begin{array}{l}\text { Particle light } \\
\text { scattering }\end{array}$ & $\begin{array}{l}\text { Optec NGN-3 ambient } \\
\text { nephelometer }\end{array}$ & CMU & $10 \mathrm{~min}$ & Continuous & $7 / 16 / 01-6 / 30 / 02$ \\
\hline $\begin{array}{l}\text { Particle } \\
\text { hygroscopicity }\end{array}$ & CMU DAASS & CMU & $1 \mathrm{~h}$ & $\begin{array}{l}\text { Semi- } \\
\text { continuous }\end{array}$ & $7 / 1-8 / 31 / 01,1 / 1-7 / 1 / 02$ \\
\hline Cloud condensation & DH Associates M1 CCN & CMU & $4 \mathrm{~h}$ & $\begin{array}{l}\text { Semi- } \\
\text { continuous }\end{array}$ & $9 / 01$ \\
\hline \multicolumn{6}{|l|}{$\begin{array}{l}\text { Single particle } \\
\text { characteristics }\end{array}$} \\
\hline $\begin{array}{l}\text { Single particle polar } \\
\text { organics }\end{array}$ & RSMS-III & $\mathrm{UCD}, \mathrm{UD}$ & $10 \mathrm{~min}$ & $\begin{array}{l}\text { Semi- } \\
\text { continuous }\end{array}$ & $9 / 20 / 01-10 / 1 / 02$ \\
\hline $\begin{array}{l}\text { Single particle ion } \\
\text { composition }\end{array}$ & RSMS-III & $\mathrm{UCD}, \mathrm{UD}$ & $10 \mathrm{~min}$ & $\begin{array}{l}\text { Semi- } \\
\text { continuous }\end{array}$ & $9 / 20 / 01-10 / 1 / 02$ \\
\hline Particle morphology & Nuclepore filter with SEM & RJL & $24 \mathrm{~h}$ & Daily & ESP01, ESP02 Intensives \\
\hline \multicolumn{6}{|l|}{ Gaseous species } \\
\hline $\begin{array}{l}\text { Light }\left(\mathrm{C}_{2}-\mathrm{C}_{12}\right) \\
\text { hydrocarbons }\end{array}$ & Canister with GC-FID analysis & CMU & $24 \mathrm{~h}$ & 3rd day & $9 / 1 / 01-7 / 31 / 02^{\mathrm{d}}$ \\
\hline $\begin{array}{l}\text { Light }\left(\mathrm{C}_{2}-\mathrm{C}_{12}\right) \\
\text { hydrocarbons }\end{array}$ & Canister with GC-FID analysis & CMU & $24 \mathrm{~h}$ & Daily & ESP02 Intensive \\
\hline All hydrocarbons & UCB GC-FID/MSD & $\mathrm{UCB}$ & $1 \mathrm{~h}$ & $\begin{array}{l}\text { Semi- } \\
\text { continuous }\end{array}$ & $1 / 9-2 / 12,7 / 10-8 / 10 / 02$ \\
\hline $\mathrm{CO}$ & API 300 infrared absorption & CMU & $10 \mathrm{~min}$ & Continuous & $7 / 1 / 01-9 / 1 / 02$ \\
\hline $\mathrm{SO}_{2}$ & API 100A pulsed fluorescence & CMU & $10 \mathrm{~min}$ & Continuous & $7 / 1 / 01-9 / 1 / 02$ \\
\hline $\mathrm{NO}$ and $\mathrm{NO}_{2}$ & API 200A chemiluminescence & $\mathrm{CMU}$ & $10 \mathrm{~min}$ & Continuous & $7 / 1 / 01-9 / 1 / 02$ \\
\hline $\mathrm{O}_{3}$ & API 400A UV absorption & CMU & $10 \mathrm{~min}$ & Continuous & $7 / 1 / 01-9 / 1 / 02$ \\
\hline $\begin{array}{l}\text { Water soluble } \\
\text { inorganic gases }\end{array}$ & $\begin{array}{l}\text { Khlystov steam sampler with } \\
\text { IC analysis }\end{array}$ & $\mathrm{CMU}$ & $1-2 \mathrm{~h}$ & $\begin{array}{l}\text { Semi- } \\
\text { continuous }\end{array}$ & $7 / 1 / 01-9 / 21 / 02$ \\
\hline Inorganic gases & CMU sampler with IC analysis & $\mathrm{CMU}$ & $24 \mathrm{~h}$ & Daily & $7 / 1 / 01-7 / 1 / 02^{\mathrm{d}}$ \\
\hline Inorganic gases & CMU sampler with IC analysis & $\mathrm{CMU}$ & $4-6 h$ & 5 per day & ESP01 Intensive \\
\hline $\begin{array}{l}\text { Total soluble } \\
\text { peroxides }\end{array}$ & CSU monitor & $\mathrm{CSU}$ & $1 \mathrm{~h}$ & Continuous & $7 / 1 / 01-8 / 18 / 02^{\mathrm{d}}$ \\
\hline
\end{tabular}


Table 5 (continued)

\begin{tabular}{llllll}
\hline Observable & Method $^{\mathrm{a}}$ & Group $^{\mathrm{b}}$ & Resolution & Frequency $^{\text {Period of operation }^{\mathrm{c}}}$ \\
\hline $\begin{array}{l}\text { Hydrogen and } \\
\text { organic peroxides }\end{array}$ & CSU monitor & CSU & $1 \mathrm{~h}$ & Continuous & ESP01, ESP02 Intensives \\
$\begin{array}{l}\text { Fog } \\
\text { Fog composition }\end{array}$ & $\begin{array}{l}\text { CASCC2 collector with IC, } \\
\text { TOC } \text { and pH analyses }\end{array}$ & CSU & Per event & $\begin{array}{l}8 \text { events } \\
\text { captured }\end{array}$ & $7 / 1 / 01-9 / 1 / 02$
\end{tabular}

\begin{tabular}{|c|c|c|c|c|c|}
\hline $\begin{array}{l}\text { Meteorology } \\
\text { Wind speed/direction }\end{array}$ & $\begin{array}{l}\text { Met One } 014 \mathrm{~A} \text { wind vane and } \\
\text { cup anemometer }\end{array}$ & $\mathrm{CMU}$ & $10 \mathrm{~min}$ & Continuous & $7 / 1 / 01-9 / 1 / 02$ \\
\hline Temperature & $\begin{array}{l}\text { Campbell HMP45C thermistor- } \\
\text { based sensor }\end{array}$ & $\mathrm{CMU}$ & $10 \mathrm{~min}$ & Continuous & $7 / 1 / 01-9 / 1 / 02$ \\
\hline Relative humidity & $\begin{array}{l}\text { Campbell HMP45C } \\
\text { capacitance thin film sensor }\end{array}$ & $\mathrm{CMU}$ & $10 \mathrm{~min}$ & Continuous & $7 / 1 / 01-9 / 1 / 02$ \\
\hline UV solar radiation & $\begin{array}{l}\text { Kipp \& Zonen CUV3 } \\
\text { broadband UV radiometer }\end{array}$ & $\mathrm{CMU}$ & $10 \mathrm{~min}$ & Continuous & $7 / 1 / 01-9 / 1 / 02$ \\
\hline Total solar radiation & $\begin{array}{l}\text { Kipp \& Zonen CM3 } \\
\text { pyranometer }\end{array}$ & $\mathrm{CMU}$ & $10 \mathrm{~min}$ & Continuous & $7 / 1 / 01-9 / 1 / 02$ \\
\hline Precipitation & Met One 370 rain gauge & CMU & $10 \mathrm{~min}$ & Continuous & $7 / 1 / 01-9 / 1 / 02$ \\
\hline Pressure & $\begin{array}{l}\text { Campbell CS105 Aneroid } \\
\text { barometer }\end{array}$ & CMU & $10 \mathrm{~min}$ & Continuous & $7 / 1 / 01-9 / 1 / 02$ \\
\hline \multicolumn{6}{|c|}{ 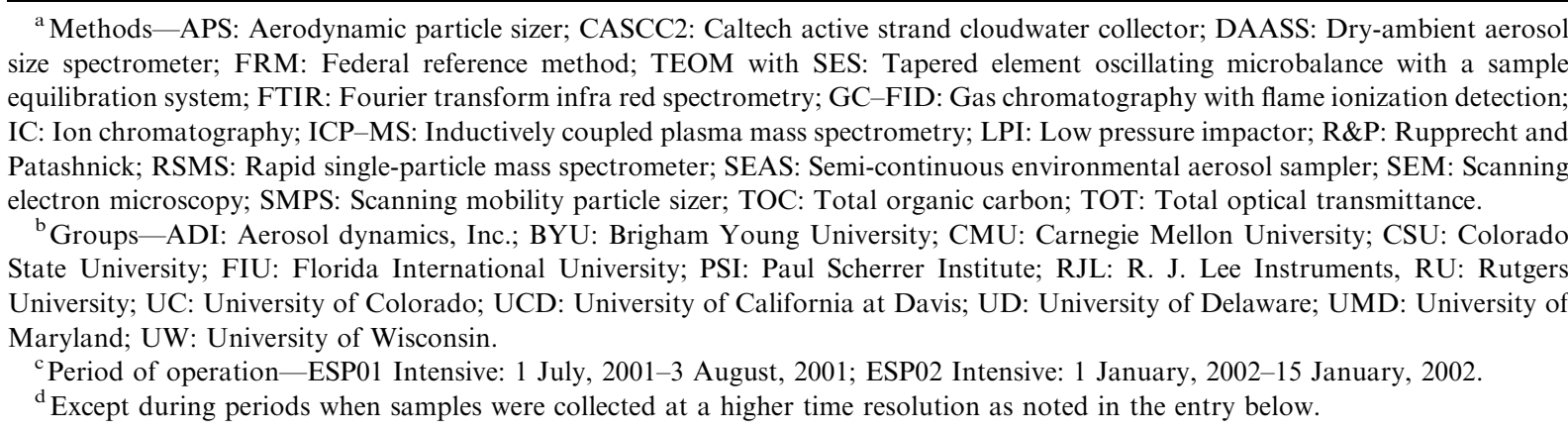 } \\
\hline
\end{tabular}

to verify the measurements are presented in detail by Khlystov et al. (2001).

During the intensive periods, $\mathrm{PM}_{2.5}$ aerosol mass, inorganic ions, organic and elemental carbon, and metal concentrations were measured at the satellite sites (Table 3). During two special experiments at the Florence PA satellite site in January and July of 2002, the $24 \mathrm{~h}$ integrated $\mathrm{PM}_{2.5}$ organic speciation and $10 \mathrm{~min}$ aerosol size distribution between 0.012 and $0.28 \mu \mathrm{m}$ was also measured. Aircraft high time resolution measurements of meteorology, aerosol properties, and gas-phase species were obtained during select days of July and August 2001 and 2002 (Table 4). The Jacko aircraft conducted 4 flights in 2 days over Pittsburgh, PA (July 23, 2001 and August 2, 2001). The Maryland aircraft conducted 10 flights in July and August of 2001 and 2002 over the western PA region.

PM mass characterization. Integrated samples of $\mathrm{PM}_{2.5}$ mass were collected onto Teflon filters using a Partisol $^{\mathrm{R}}$-Federal Reference Method (FRM) Model
$2000 \mathrm{PM}_{2.5}$ Air Sampler (Rupprecht \& Patashnick, Albany, NY). Integrated samples of $\mathrm{PM}_{2.5}$ and $\mathrm{PM}_{10}$ mass were also collected on Teflon filters using a Dichotomous (Dichot) sampler (Thermo Andersen, Model 241). Both samplers were operated using standard procedures (U.S.E.P.A., 1998). A MicroOrifice Uniform Deposit Impactor (MOUDI) (MSP Corp., Model 110) was used to collect size-resolved samples of particles on Teflon substrates using standard procedures (MSP Corp., 1998). This permitted estimates of concentrations of particles, $\mathrm{PM}_{x}$, with diameters less than $2.5,1.8,1.0,0.56,0.32,0.18,0.10$, and $0.056 \mu \mathrm{m}$. The FRM, Dichot, and MOUDI filters were analyzed for mass concentration by gravimetric analysis using standard protocols (Code of Federal Regulations, 2002) in a humidity-controlled equilibration chamber in the CMU Air Quality Laboratory.

Semi-continuous $\mathrm{PM}_{2.5}$ mass was measured using a Tapered Element Oscillating Microbalance (TEOM) monitor (Rupprecht and Patashnick, Model 1400A). 
The Sample Equilibration System (SES) permitted particles to be collected onto the Pallflex tapered element at temperature of $30^{\circ} \mathrm{C}$.

$P M$ size distributions. A suite of particle sizing instruments was used to semi-continuously measure the number size distributions for particles from 0.003 to $10 \mu \mathrm{m}$ (Stanier et al., 2004a). The suite included a nanoScanning Mobility Particle Sizer (SMPS) (TSI, Inc., Model 3936N25), a standard SMPS (TSI, Inc., Model 3936L10), and an Aerodynamic Particle Sizer (APS) (TSI, Inc., Model 3320). The 3 separate distributions were combined, after inversion, by using the nanoSMPS data up to $0.03 \mu \mathrm{m}$, the SMPS data from 0.03 to $0.6 \mu \mathrm{m}$, and the APS data beginning at $0.6 \mu \mathrm{m}$. Aerodynamic diameters measured by the APS were converted to mobility diameters prior to combining data using an effective density and a least-squares fitting algorithm (Khlystov et al., 2003). The average effective density used for the period from July to August of 2001 was $1.52 \mathrm{~g} \mathrm{~cm}^{-3}$, with 5 th and 95th percentile values of 0.94 and $1.94 \mathrm{~g} \mathrm{~cm}^{-3}$. Effective density was used over a density derived from speciated aerosol measurements for the following three reasons: $\mathrm{PM}_{2.5}$ speciation measurements were not always available; the density derived from speciated data requires an estimate of water content, which was not always available; and the effective density takes into account the unknown shape factor.

$P M$ inorganic ions. Integrated samples of $\mathrm{PM}_{10}$ and $\mathrm{PM}_{2.5}$ were drawn through cyclones and denuders and collected onto filter packs of PTFE Teflon, nylon, and cellulose-fiber filters (Takahama et al., 2004). Size resolved $\mathrm{PM}_{x}$ inorganic ion samples were collected onto Teflon substrates using a MOUDI. The filter pack and MOUDI samples were analyzed for nitrate, sulfate, ammonium and chloride by Ion Chromatography (IC) using procedures similar to those described by Jaffrezo et al. (1998).

Semi-continuous measurements of total nitrate $\left(\mathrm{PM}_{2.5}\right.$ nitrate plus $\left.\mathrm{HNO}_{3}\right)$, total sulfate $\left(\mathrm{PM}_{2.5}\right.$ sulfate plus $\left.\mathrm{SO}_{2}\right)$, and total ammonium $\left(\mathrm{PM}_{2.5}\right.$ ammonium plus $\mathrm{NH}_{3}$ ) were made using a steam sampler (Khlystov et al., 1995) with analysis by IC. Total ammonium was measured continuously on-line using the steam sampler and the detector of Slanina et al. (2001). Comparisons of steam sampler measurements against denuder-filter pack measurements did not reveal an ammonium artifact, consistent with the findings of Slanina et al. (2001), Erisman et al. (2001), and Zhang et al. (2002).

Semi-continuous measurements of $\mathrm{PM}_{2.5}$ nitrate and sulfate were made using Integrated Collection and Vaporization Cell (ICVC) instruments (Rupprecht and Patashnick, Models 8400 S and $8400 \mathrm{~N}$ ) and processed using the method described by Wittig et al. (2003).

PM Trace Metals. Integrated $\mathrm{PM}_{10}$ and $\mathrm{PM}_{2.5}$ samples were collected on cellulose filters using Thermo
Andersen High-Volume (High Vol) samplers operated using standard procedures (Thermo Andersen, 1999; U.S.E.P.A., 1999). The filters were digested in a combination of nitric acid, hydrofluoric acid, and hydrogen peroxide in a sealed microwave vessel and analyzed according to standard procedures by Inductively Coupled Plasma Mass Spectrometry (ICP-MS) (U.S.E.P.A., 1991; U.S.E.P.A., 1994) to determine concentrations of $\mathrm{Al}$, As, $\mathrm{Ba}, \mathrm{Ca}, \mathrm{Cd}, \mathrm{Cr}, \mathrm{Cu}, \mathrm{Fe}, \mathrm{Ga}, \mathrm{K}, \mathrm{Mg}, \mathrm{Mn}, \mathrm{Mo}, \mathrm{Na}, \mathrm{Ni}, \mathrm{Pb}$, $\mathrm{Rb}, \mathrm{Sb}, \mathrm{Se}, \mathrm{Sr}, \mathrm{Ti}, \mathrm{V}$ and $\mathrm{Zn}$.

Semi-continuous measurements of 11 metals (Al, As, $\mathrm{Cd}, \mathrm{Cr}, \mathrm{Cu}, \mathrm{Fe}, \mathrm{Mn}, \mathrm{Ni}, \mathrm{Pb}, \mathrm{Se}$ and $\mathrm{Zn}$ ) were also made using the Semi-continuous Environmental Aerosol Sampler (SEAS) (Kidwell and Ondov, 2001) with offline analysis using Graphite Furnace Atomic Absorption (GFAA).

$P M$ carbonaceous material. Integrated samples of $\mathrm{PM}_{2.5}$ were collected on bare quartz filters and two backup quartz filters, one downstream of the bare quartz filter and the other in a parallel port behind a Teflon filter (Subramanian et al., 2003). Integrated samples of $\mathrm{PM}_{2.5}$ were also denuded and collected on quartz fiber filters with a carbon impregnated graphite backup filter (Subramanian et al., 2003). Size resolved $\mathrm{PM}_{x}$ samples were collected on ungreased foil substrates using a MOUDI sampler. The quartz and foil filters were analyzed for organic carbon (OC) and elemental carbon (EC) using the Thermal Optical Transmittance (TOT) method and the NIOSH thermal evolution protocol (NIOSH, 1996; Cabada et al., 2004a) in the CMU Air Quality Laboratory. Integrated samples of $\mathrm{PM}_{2.5}$ OC and EC were also collected using the PCBOSS diffusion denuder sampler (Eatough et al., 2001) and analyzed at Brigham Young University.

Additional size-resolved samples were collected on zinc selenide disks using a Hering Low Pressure Impactor (LPI) sampler (Aerosol Dynamics Inc.) and analyzed for organic functional groups using Fourier Transform Infrared Spectroscopy (FTIR) at Rutgers University (Blando et al., 2001).

Semi-continuous measurements of $\mathrm{PM}_{2.5} \mathrm{OC}$ and EC were conducted using the Sunset Laboratory in situ carbon analyzer with a denuder placed upstream of the instrument (Turpin et al., 1990). Semi-continuous measurements of $\mathrm{PM}_{2.5}$ total carbon were obtained using an the Aerosol Dynamics ICVC total carbon instrument which is similar in design to the R\&P instruments used to measure $\mathrm{PM}_{2.5}$ nitrate and sulfate semi-continuously (Stolzenburg and Hering, 2000).

PM biological material. Ambient bioaerosols were collected using a High Vol sampler for July 2001, and using a low volume sampler for all other periods. The samples were analyzed at Colorado State University using direct epi-fluorescent microscopy and newer molecular biology methods to obtain microbioaerosol concentrations of bacteria, fungi, and their spores. 
PM hygroscopicity. The ability of ambient fine particles to absorb water and grow was measured using the DryAmbient Aerosol Size Spectrometer (DAASS) (Stanier et al., 2004a). The water content of aerosols from 0.003 to $10 \mu \mathrm{m}$ was computed as the difference in particle volume distributions at ambient $\mathrm{RH}$ and at 'dried' conditions. Dried conditions $(\mathrm{RH}<30 \%)$ were achieved by sampling ambient air though a system of Nafion dryers prior to analysis by the suite of particle sizing instruments. Sizedependent losses across the DAASS inlet were 17\% at $2.5 \mu \mathrm{m}$ and practically zero at $1 \mu \mathrm{m}$, determined as the difference in total particle counts across the dried and ambient RH inlets when a dry monodisperse ammonium sulfate was injected into both inlets. Particle losses below $20 \mathrm{~nm}$ were estimated using the empirical particle loss correlation of Willeke and Baron (1993).

Cloud Condensation Nuclei (CCN) concentration was measured using CCN counter (DH Associates, Model M1). The CCN concentration is directly related to the ability of the particles to become cloud droplets.

Aerosol light scattering. Continuous $\mathrm{PM}_{2.5}$ scattering coefficients from $5^{\circ}$ to $175^{\circ}$ at a wavelength of $550 \mathrm{~nm}$ were measured at ambient conditions using an integrating nephelometer (Optec, Inc., NGN-3) with a $\mathrm{PM}_{2.5}$ cyclone at the inlet. Temperature was measured at the inlet to the nephelometer and inside the nephelometer at the chamber outlet. Comparisons between these two measurements were used to correct for the decrease in relative humidity of the sampled air due to heating inside the chamber.

Aerosol mass spectroscopy. The Aerodyne Mass Spectrometer (AMS) was used to measure size resolved aerosol chemical composition semi-continuously (Jayne et al., 2000; Jimenez et al., 2003). The instrument determined the size distributions from 0.050 to $1 \mu \mathrm{m}$ of sulfate, nitrate, ammonium, chloride, total organics, and water chemical components.

Single particle mass spectroscopy. The Rapid Single Particle Mass Spectrometer (RSMS-III) measured the size and composition of individual particles for nine particle size ranges between 0.04 and $2 \mu \mathrm{m}$ (Carson et al.,
1997; Ge et al., 1998). This method was used to detect metals and metal oxides, refractory crustal materials, sulfates and nitrates, aromatic and amine organic compounds, and elemental carbon in single particles.

Single particle SEM analyses. $\mathrm{PM}_{10}$ and $\mathrm{PM}_{2.5}$ aerosol samples were collected onto Nuclepore filters for offsite analysis by Scanning Electron Microscopy (SEM) at the laboratories of R.J. Lee, Inc. The SEM analyses provided elemental composition and morphology of individual particles, and were used to identify bioaerosols, soot, and spherical aluminosilicate particles.

Gaseous species. Integrated VOC samples were collected in 61 fused silica-lined canisters (Entech Instruments, Part 29-10621) using a passive inlet (Entech Instruments, Model CS1200) and analyzed in the CMU Air Quality Laboratory using standard GC-FID techniques (Lewis et al., 1999). This approach permitted the quantification of over 70 compounds, including some of the standard set of Photochemical Assessment Monitoring Station (PAMS) species.

Semi-continuous VOC measurements were made by the UC Berkeley group using an in-situ two-channel GC/MS/FID system. The channels were configured with different preconditioning systems, preconcentration traps, and chromatography columns, enabling quantification of over 60 compounds, including C3-C6 alkanes, alkenes, alkynes, and aromatic, oxygenated and halogenated compounds.

Continuous measurements of $\mathrm{O}_{3}, \mathrm{NO}$ and $\mathrm{NO}_{2}, \mathrm{CO}$, and $\mathrm{SO}_{2}$ gas concentrations were made using highsensitivity gas analyzers (Advanced Pollution Instrumentation, respective Models 400A, 200A, 300 and 100A). Continuous measurements of organic peroxide, hydrogen peroxide, and total soluble peroxide were made using the CSU monitor based on the method of Lazrus et al. (1986).

Fog. Fog composition was measured using a compact version of the Caltech Active Strand Cloudwater Collector known as the CASCC2 (Demoz et al., 1996). Eight major fog events were captured during the 14month baseline study period and are listed in Table 6 .

Table 6

Days during which fog was collected at the central site

\begin{tabular}{|c|c|c|c|c|}
\hline Start date (EST) & Start hour (EST) & End date (EST) & End hour (EST) & $\begin{array}{l}\text { Liquid collected during } \\
\text { event }(\mathrm{ml})\end{array}$ \\
\hline $2001 / 08 / 10$ & $00: 15$ & $2001 / 08 / 10$ & $06: 50$ & 21.5 \\
\hline $2001 / 08 / 26$ & $18: 20$ & $2001 / 08 / 27$ & 06:10 & 88.2 \\
\hline $2001 / 11 / 02$ & $16: 00$ & $2001 / 11 / 02$ & $21: 20$ & 27.8 \\
\hline $2001 / 11 / 17$ & $03: 15$ & $2001 / 11 / 17$ & $07: 45$ & 36.8 \\
\hline $2001 / 11 / 17$ & 19:05 & $2001 / 11 / 18$ & $08: 10$ & 444.5 \\
\hline $2001 / 11 / 19$ & $18: 30$ & $2001 / 11 / 19$ & $19: 35$ & 24.0 \\
\hline $2002 / 05 / 07$ & $09: 50$ & $2002 / 05 / 08$ & $23: 45$ & 19.4 \\
\hline $2002 / 05 / 11$ & $22: 45$ & $2002 / 05 / 13$ & $11: 05$ & 18.6 \\
\hline
\end{tabular}


These fog samples were analyzed onsite for $\mathrm{pH}$. Aliquots of the collected fog were also prepared and analyzed offsite for major anions and cations using IC, and total organic carbon (TOC) and dissolved organic carbon (DOC) using a TOC analyzer (Shimadzu, Model 5000-A).

Meteorology. Precipitation, temperature, relative humidity, wind speed and direction, and UV intensity were measured using standard methods. Backward air mass trajectories were calculated using the methods of Harris and Kahl (1994).

\section{PAQS Source sampling experiments}

Many primary sources of atmospheric aerosols for the Pittsburgh region are inadequately characterized. Several of these sources have been selected for more complete characterization: vehicle exhaust (truck and diesel), coal-fired boilers, coke production, dust (soil and road), vegetative detritus, and fireplaces (waste wood). Field studies that include stack testing using the CMU dilution sampler (Lipsky et al., 2002), fence line monitoring with high time resolution instruments, and

24-hour
$\mathrm{PM}_{2.5}$ mass
$\left(\mu \mathrm{g} / \mathrm{m}^{3}\right)$

24-hour $\mathrm{PM}_{2.5}$ sulfate $\left(\mu \mathrm{g} / \mathrm{m}^{3}\right)$

24-hour $\mathrm{PM}_{2.5}$ nitrate $\left(\mu \mathrm{g} / \mathrm{m}^{3}\right)$

24-hour
$\mathrm{PM}_{2.5}$
organic
carbon
$\left(\mu \mathrm{g}-\mathrm{C} / \mathrm{m}^{3}\right)$

24-hour

$\mathrm{PM}_{2.5}$

elemental

carbon

$\left(\mu \mathrm{g}-\mathrm{C} / \mathrm{m}^{3}\right)$
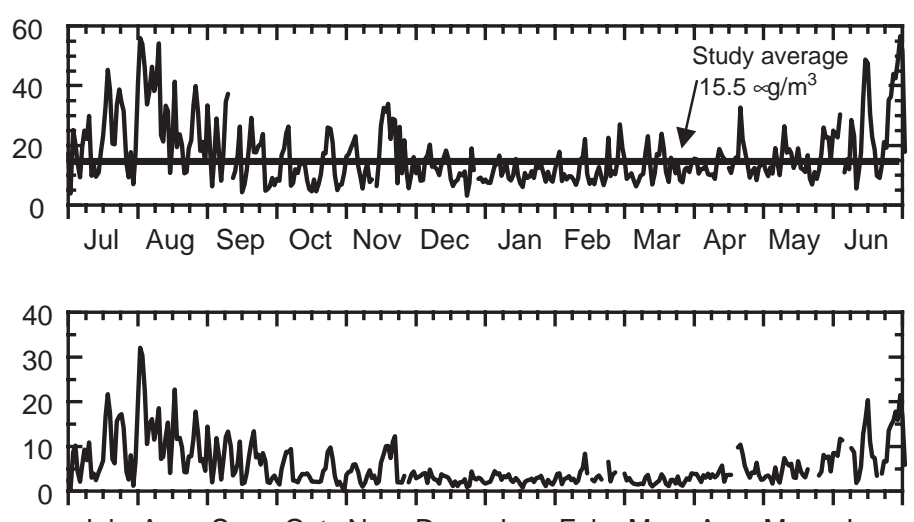

Jul Aug Sep Oct Nov Dec Jan Feb Mar Apr May Jun

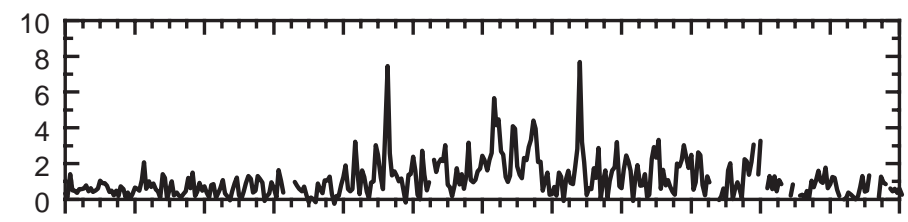

Jul Aug Sep Oct Nov Dec Jan Feb Mar Apr May Jun
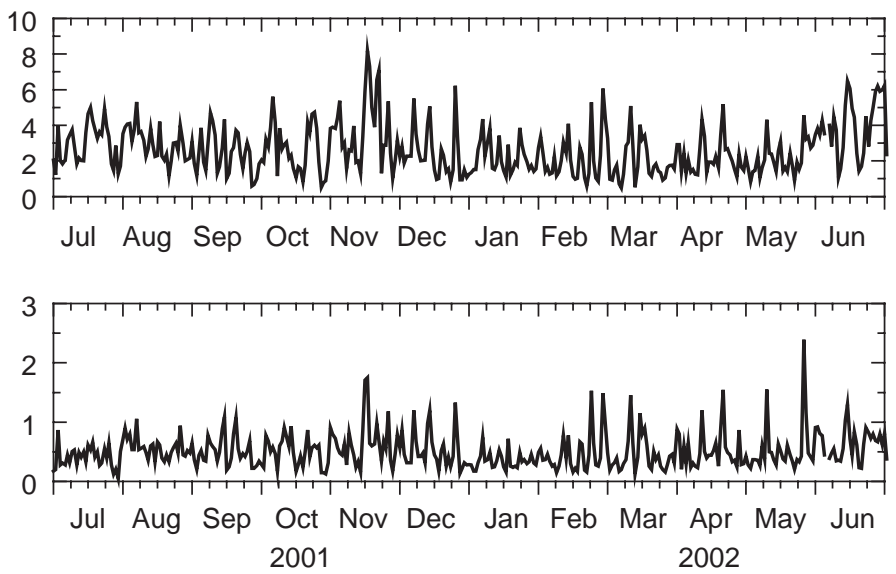

Fig. 3. Daily $24 \mathrm{~h}$ average mass, sulfate, nitrate, organic carbon, and elemental carbon concentrations at the central site from July 2001 to June 2002. Mass concentrations are measured using a TEOM. Organic carbon concentrations are based on the difference between the OC collected on the upstream ('bare quartz') and downstream quartz filters of the CMU TQQQ sampler double-quartz filter pack. Elemental carbon concentrations are based on the TQQQ upstream quartz filter alone. Nitrate and sulfate concentrations are composited from measurements made using the CMU inorganic sampler, the PC-BOSS, the steam sampler, and the Rupprecht and Patashnick 8400 instruments. 
sampling in a highway tunnel are being performed to develop updated emission profiles for these source categories. The source characterization uses the same approaches and instrumentation as the central site ambient monitoring, including detailed organic speciation and single-particle mass spectrometry. These source profiles will be compared to ambient measurements by compiling source activity data and by using a variety of source-receptor techniques.

\section{Daily aerosol measurements collected at the central site}

The study average $\mathrm{PM}_{2.5}$ mass concentration was $15.5 \mu \mathrm{g} \mathrm{m}^{-3}$. Average $24 \mathrm{~h}$ concentrations of mass, sulfate, nitrate, organic carbon, and elemental carbon are presented in Fig. 3. On average, all species exhibited day to day variations in concentration. For example, in July 2001, the maximum and minimum $24 \mathrm{~h}$ average concentrations were 60.9 and $3.7 \mu \mathrm{g} \mathrm{m}^{-3}\left(\mathrm{PM}_{2.5}\right.$ mass), 33.7 and $0.1 \mu \mathrm{g} \mathrm{m}^{-3}\left(\mathrm{PM}_{2.5}\right.$ sulfate), 2.5 and $0.0 \mu \mathrm{g} \mathrm{m}^{-3}$ $\left(\mathrm{PM}_{2.5}\right.$ nitrate), 11.4 and $1.2 \mu \mathrm{g} \mathrm{m}^{-3}\left(\mathrm{PM}_{2.5}\right.$ organic carbon), and 1.7 and $0.1 \mu \mathrm{g} \mathrm{m}^{-3} \quad\left(\mathrm{PM}_{2.5}\right.$ elemental carbon).

The $24 \mathrm{~h} \mathrm{PM}_{2.5}$ Federal Standard of $65 \mu \mathrm{g} \mathrm{m}{ }^{-3}$ was never exceeded during the study at the PAQS central site. However, on the nine days shown in Table 7, the $24 \mathrm{~h}$ average $\mathrm{PM}_{2.5}$ mass concentration was greater than $50 \mathrm{\mu g} \mathrm{m}^{-3}$. Six of these nine days were consecutive 2-day episodes. All 9 days occurred during the summer months of 2001 and 2002, when the $24 \mathrm{~h}$ average ambient temperature was higher than average. On three of the days, the episodes were associated with higher than average sulfate and lower than average organic carbon concentrations ( 1 and 2 August, 2001, and 18 July, 2002). On four other days, the episodes were associated with lower than average sulfate and higher than average organic carbon concentrations (26 June, 1 and 2 July, and 8 July, 2002).

In the remaining sections, seasonality in meteorology, gas-phase and aerosol-phase measurements is assessed using monthly average measurements. This approach allowed seasonal generalizations to be made that were not biased by inconsistencies observed during anomalous months, such as July 2001 and November 2001.

\section{Seasonal summary of measurements collected at the central site}

Meteorology. The seasonal variations in meteorological variables are shown in Fig. 4. Monthly average ambient relative humidity $(\mathrm{RH})$ did not exhibit a strong seasonal dependence and was approximately 60-65\%. On average, the predominant wind direction was
Table 7

Days on which the $24 \mathrm{~h}$ average $\mathrm{PM}_{2.5}$ mass was greater than $50 \mu \mathrm{g} \mathrm{m}^{-3}$

\begin{tabular}{lll}
\hline Date & $\begin{array}{l}\text { Maximum 1 h mass } \\
\text { concentration }\left(\mu \mathrm{g} / \mathrm{m}^{3}\right)\end{array}$ & $\begin{array}{l}\text { Average 24h mass } \\
\text { concentration }\left(\mu \mathrm{g} / \mathrm{m}^{3}\right)\end{array}$ \\
\hline $8 / 1 / 01$ & 63.8 & 55.9 \\
$8 / 2 / 01$ & 75.0 & 54.0 \\
$8 / 9 / 01$ & 72.6 & 54.2 \\
$6 / 25 / 02$ & 75.4 & 56.6 \\
$6 / 26 / 02$ & 74.2 & 51.5 \\
$7 / 1 / 02$ & 76.7 & 60.9 \\
$7 / 2 / 02$ & 79.5 & 56.1 \\
$7 / 8 / 02$ & 69.3 & 51.1 \\
$7 / 18 / 02$ & 70.3 & 53.3 \\
\hline
\end{tabular}

southeasterly or northwesterly in July 2001 and southwesterly in January 2002. The maximum wind speeds were higher, on average, in January 2002 than in July 2001.

$\mathrm{PM}_{10}$ and $\mathrm{PM}_{2.5}$ mass concentrations. Monthly average $\mathrm{PM}_{10}$ and $\mathrm{PM}_{2.5}$ mass concentrations are presented in Fig. 5. On average, approximately $65 \%$ of the $\mathrm{PM}_{10}$ mass measured at PAQS was in the fine $(<2.5 \mu \mathrm{m})$ particle range. The monthly average $\mathrm{PM}_{2.5}$ mass concentrations were robust, as four independent methods of measurement (MOUDI, FRM, Dichot, and TEOM with SES) were in close agreement $\left(R^{2}>0.95\right)$ throughout the study period (Wittig and Davidson, 2003; Cabada et al., 2004b). Fig. 6 illustrates this correlation between the FRM, Dichot, and TEOM with SES but points out that the TEOM slightly overestimated the $\mathrm{PM}_{2.5}$ mass.

$\mathrm{PM}_{2.5}$ composition. Seasonal differences in the average $\mathrm{PM}_{2.5}$ mass composition are presented in Fig. 7. Organic matter concentrations for July 2001 are based on $24 \mathrm{~h}$ integrated denuder sampler measurements and a multiplier of 1.8. For the rest of the study, organic matter concentrations are based on the difference between the OC collected on the upstream ('bare quartz') and downstream quartz filters of the CMU TQQQ sampler double-quartz filter pack. Sulfate, nitrate, and ammonium concentrations are composites of measurements made from several methods, including the CMU inorganic sampler, the PC-BOSS, the steam sampler and the Rupprecht and Patashnick 8400 instruments. $\mathrm{PM}_{2.5}$ crustal material concentrations were estimated from daily $24 \mathrm{~h} \mathrm{PM}_{2.5}$ metal species measurements at the PAQS satellite sites in July 2001 using the sum of oxides algorithm (Malm et al., 1994a, b). The $\mathrm{PM}_{2.5}$ metal species concentrations did not vary substantially across the satellite sites and the estimate of $\mathrm{PM}_{2.5}$ crustal material concentration of $\sim 1 \mu \mathrm{g} \mathrm{m}^{-3}$ compared well with estimates from similar studies (Tolocka et al., 2001). Because the Pittsburgh aerosol is of a regional 

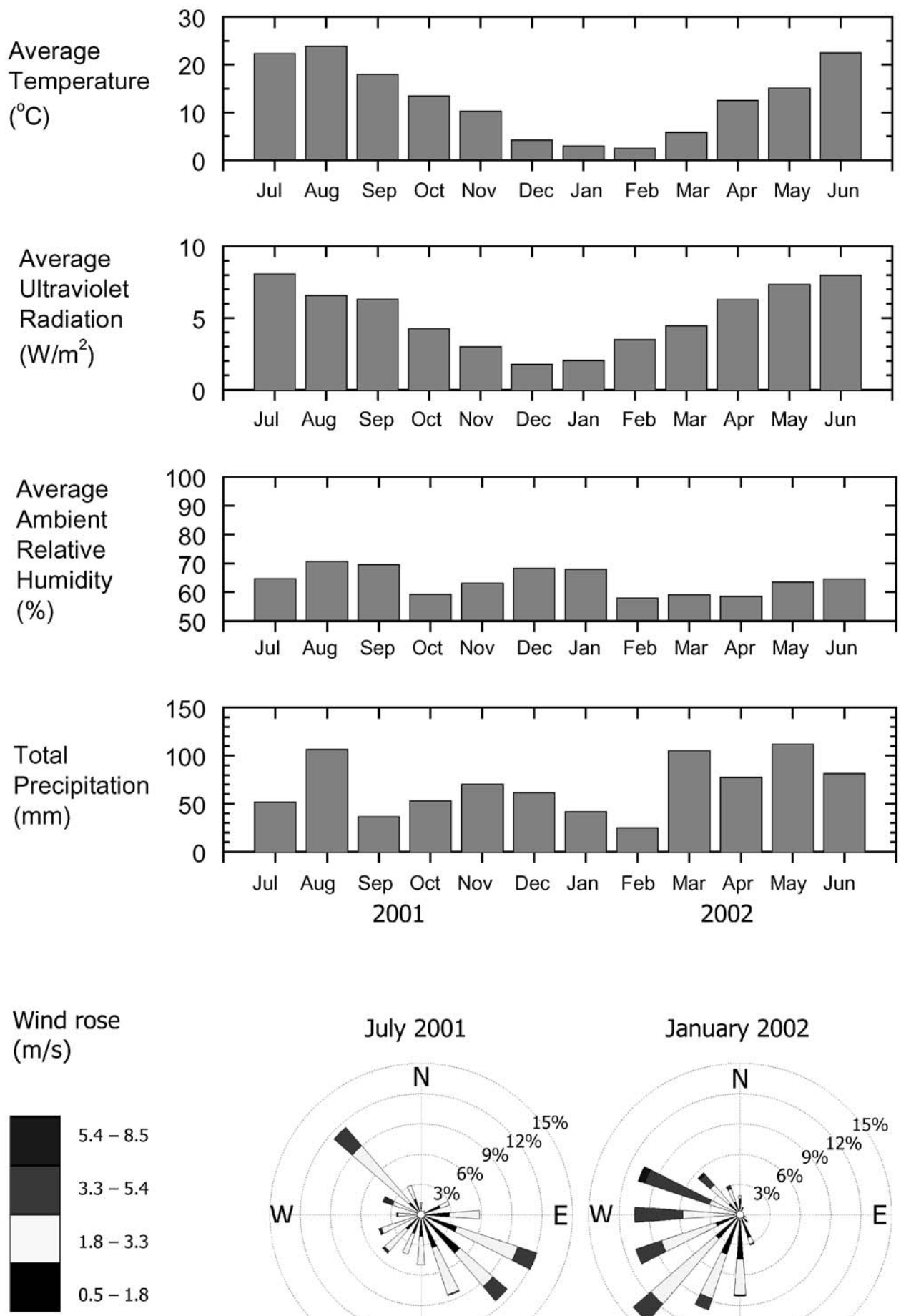

July 2001

$\mathrm{N}$

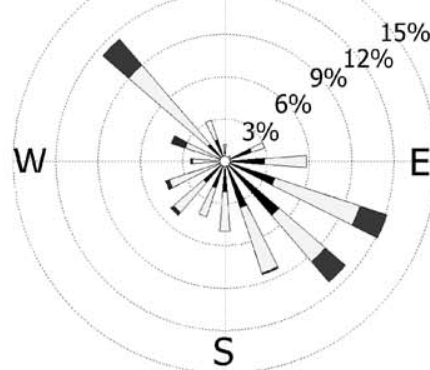

January 2002

N

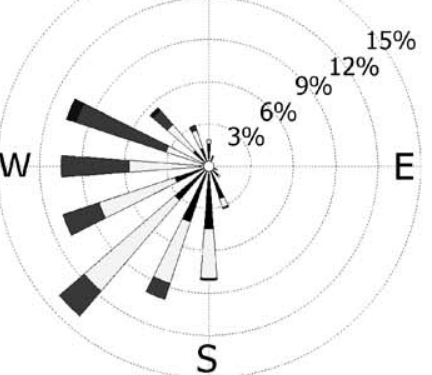

Fig. 4. Monthly average ambient temperature, ultraviolet radiation, and relative humidity, and monthly total precipitation measured at the central site from July 2001 to June 2002. Also shown is the relative occurrence of $1 \mathrm{~h}$ wind speeds for July 2001 and January 2002. 


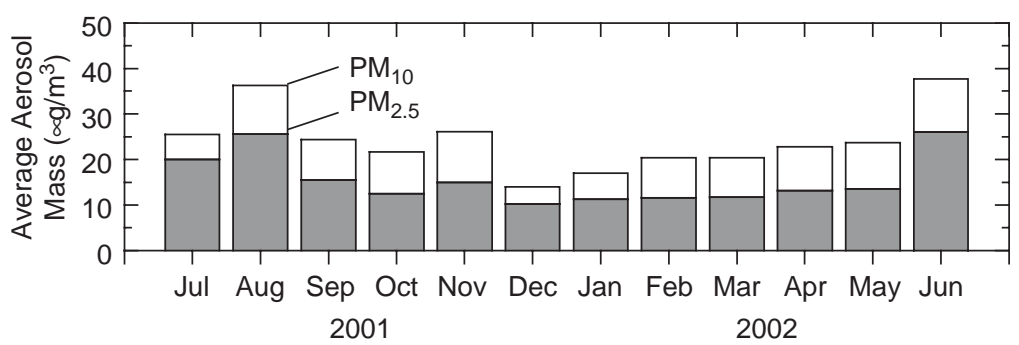

Fig. 5. Monthly average $\mathrm{PM}_{10}$ mass and $\mathbf{P M}_{2.5}$ mass measured at the central site from July 2001 to June 2002 . PM 10 mass was measured on a $24 \mathrm{~h}$ basis using the Dichot sampler, while $\mathrm{PM}_{2.5}$ mass was measured on a $24 \mathrm{~h}$ basis using the FRM sampler.

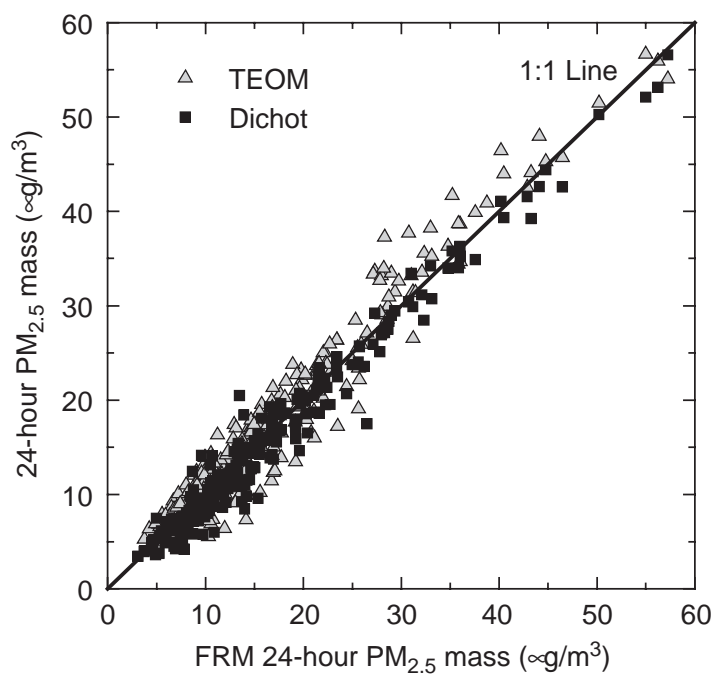

Fig. 6. Comparison of $24 \mathrm{~h}$ integrated $\mathrm{FRM} \mathrm{PM}_{2.5}$ mass measurements and other $\mathrm{PM}_{2.5}$ mass measurements collected at the central site from July 1, 2001 to June 30, 2002. Integrated Dichot measurements were collected on a daily basis, while TEOM measurements were collected continuously and averaged on a $24 \mathrm{~h}$ basis.

nature, this estimate was also used for the months of August 2001 through June 2002. Overall, the monthly average elemental and organic carbon concentrations were variable over the year, while the nitrate concentrations were highest during the winter months, and the mass and sulfate concentrations were highest during the summer months.

Although not noted in Fig. 7, seasonal variations in ultra fine aerosol composition (less than $0.1 \mu \mathrm{m}$ ), which comprise approximately $5 \%$ of the total $\mathrm{PM}_{2.5}$ mass, were also observed at the PAQS site. During the summer intensive, Cabada et al. (2004c) found that on average, $55 \%$ of the ultra fine aerosol mass was inorganic (mainly sulfate and ammonium) and $45 \%$ of the mass was carbonaceous. During the winter intensive, only $33 \%$ of

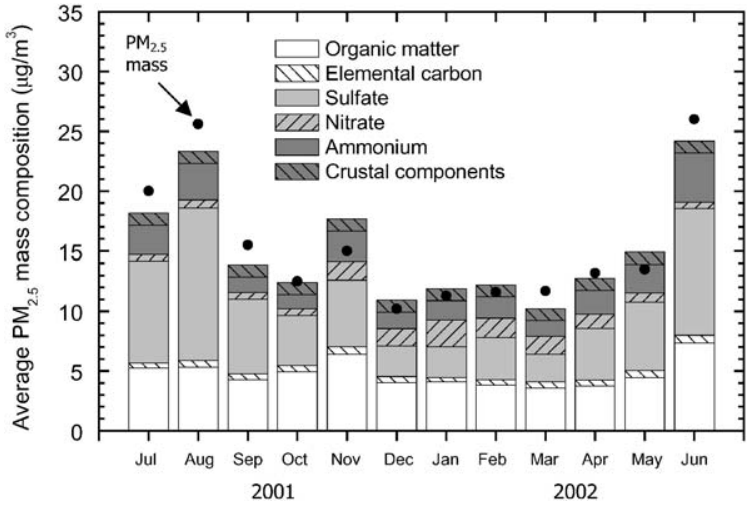

Fig. 7. $\mathrm{PM}_{2.5}$ composition on a monthly average basis at the central site from July 2001 to June 2002. Also shown is the $\mathrm{PM}_{2.5}$ total mass measured using the FRM. Organic matter concentrations for July 2001 are based on $24 \mathrm{~h}$ integrated denuder sampler measurements and the experimentally determined multiplier of 1.8. For other periods, organic matter concentrations are based on the difference between the OC collected on the upstream ('bare quartz') and downstream quartz filters of the CMU TQQQ sampler double-quartz filter pack. Sulfate, nitrate, and ammonium concentrations are composites of measurements made from several methods, including the CMU inorganic sampler, the PC-BOSS, the steam sampler and the Rupprecht and Patashnick 8400 instruments. Crustal component concentrations for August 2001 through June 2002 were estimated from daily July 2001 measurements collected at the PAQS satellite sites to be approximately $1 \mathrm{\mu g} \mathrm{m}^{-3}$.

the ultra fine aerosol mass was inorganic while $65 \%$ was carbonaceous in nature.

$P M$ size distributions. Average surface area and volume size distributions are presented in Fig. 8 for selected months. The aerosol surface area was centered at approximately $0.2 \mu \mathrm{m}$ in aerodynamic diameter. The aerosol volume distribution was bimodal, with a majority of the aerosol volume in the fine mode. The two modes were centered at approximately 0.3 and $3 \mu \mathrm{m}$. 

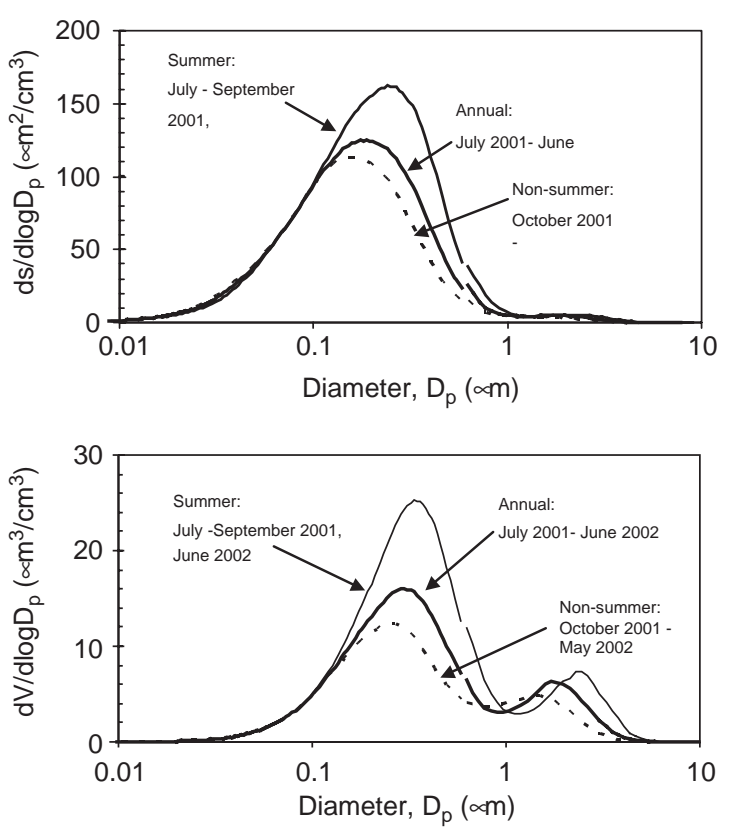

Fig. 8. Monthly average aerosol surface area and volume concentration distribution at the central site from June 2001 to May 2002, as a function of electrical mobility diameter.

\section{Seasonal differences in diurnal patterns at the central site}

Meteorology. Monthly average diurnal profiles were compiled for particular high time resolution measurements for the two intensive periods, July 2001 and January 2002. Diurnal profiles of several key meteorological variables are presented in Fig. 9. Greater than $90 \%$ and $55 \%$ of the days followed these average diurnal patterns in July 2001 and January 2002, respectively.

$\mathrm{PM}_{2.5}$ mass concentration, light scattering, and size distribution. The average diurnal profiles for $\mathrm{PM}_{2.5}$ mass concentration (Fig. 10) did not exhibit a clear diurnal trend. Moreover, less than $25 \%$ of the days followed the corresponding generalized patterns in either month. This behavior can be explained by the fact that the dominant components of the particulate mass (sulfate and organics) were not produced locally but were transported to the area (Tang et al., 2004). The light scattering at ambient RH by aerosols with diameters less than or equal to $2.5 \mu \mathrm{m}$ exhibits a diurnal pattern following that of $\mathrm{RH}$ that is more pronounced during July 2001.

Differences in the diurnal profiles of ambient and dried RH particle size and volume distributions for the intensive months are illustrated in Fig. 11. The number size distributions exhibit diurnal profiles due to diurnal variations in the ultrafine particles, with a maximum

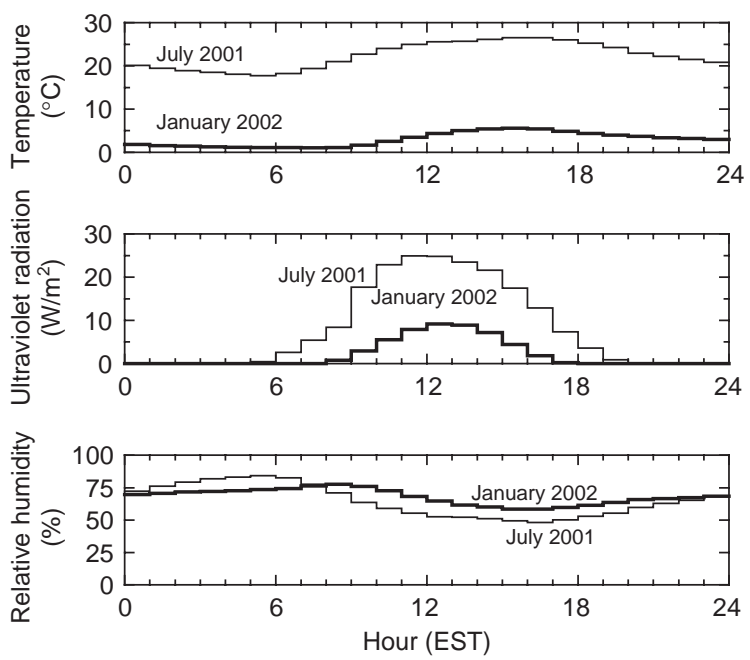

Fig. 9. Average diurnal variation in ambient temperature, ultraviolet radiation, and relative humidity for July 2001 and January 2002 at the central site.
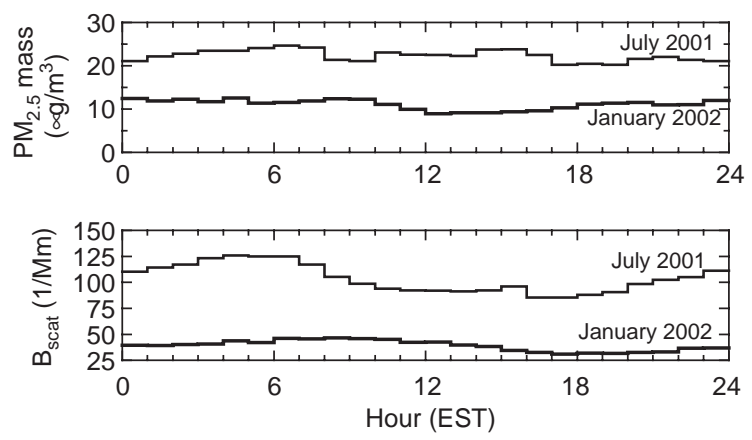

Fig. 10. Diurnal variation in $\mathrm{PM}_{2.5}$ mass and light scattering for July 2001 and January 2002 at the central site. $\mathbf{P M}_{2.5}$ mass was measured using the TEOM and back scatter was measured using the nephelometer.

total number count at midday (Stanier et al., 2004b). The difference between the maximum and minimum number count is more pronounced in the July 2001 profile, when average ambient $\mathrm{PM}_{2.5}$ concentration was higher. Approximately $40 \%$ and $55 \%$ of the days followed these patterns in July 2001 and January 2002, respectively. The dramatic difference between the July 2001 dried and ambient RH volume distributions is due to the substantial water content of the aerosols, attributed to seasonal differences in aerosol acidity.

$P M_{2.5}$ mass composition. Diurnal profiles for specific components of the $\mathrm{PM}_{2.5}$ mass were most evident for nitrate (Fig. 12). Ambient nitrate exhibited a consistent diurnal pattern throughout the study period, with maximum nitrate observed in the early morning and minimum nitrate observed in the late afternoon (Wittig 

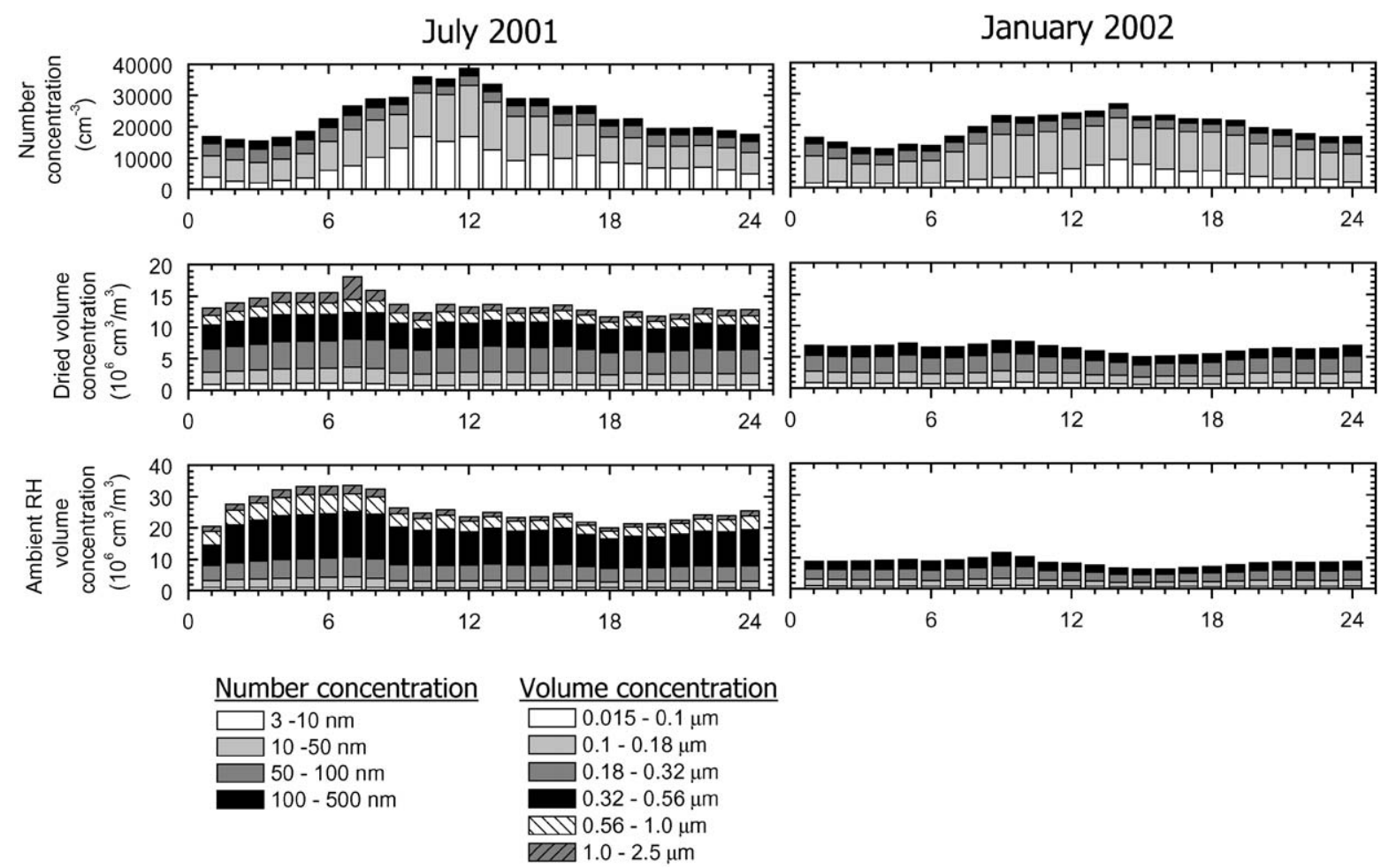

Fig. 11. Average diurnal variation in particle number, and dried and ambient RH volume concentration for July 2001 and January 2002 at the central site. Size distributions were measured using the DAASS and presented as a function of electrical mobility diameter.
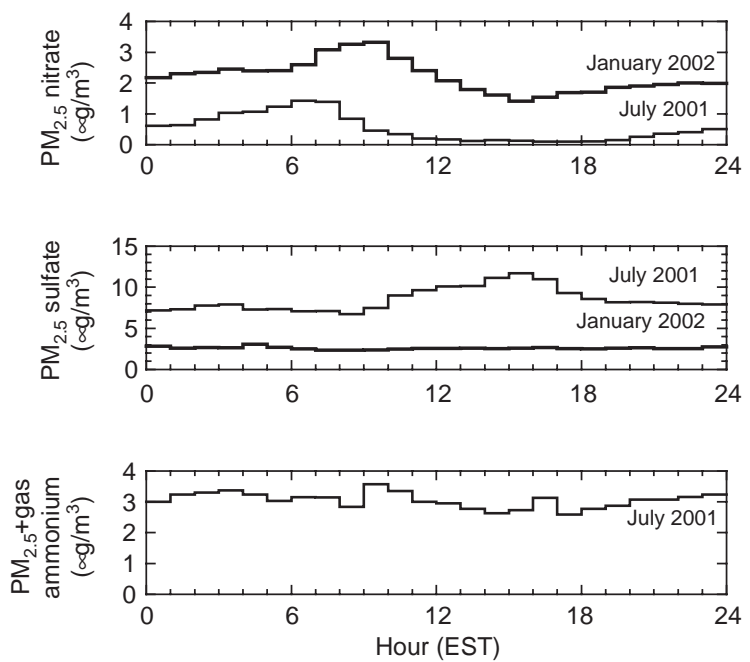

Fig. 12. Average diurnal variation in $\mathrm{PM}_{2.5}$ nitrate, sulfate, and total $\left(\mathrm{PM}_{2.5}\right.$ plus gas) ammonium for July 2001 and January 2002 at the central site. Diurnal values were based on a composite of measurements obtained using the R\&P 8400 instruments, the steam sampler, and the CMU inorganic sampler. et al., 2003). The time of the maximum and minimum nitrate shifted with seasonal changes in ambient temperature and UV radiation. Approximately $90 \%$ and $80 \%$ of the days followed the nitrate patterns in July 2001 and January 2002, respectively. Sulfate concentrations varied diurnally only during the summer, consistent with the gas-phase photochemical production during the day. During the summer, maximum sulfate was observed a couple hours before sunset. Approximately $70 \%$ of the days followed the sulfate pattern in July 2001. During the winter, sulfate concentrations were relatively stable over the course of a day on average. Diurnal profiles for were also evident for $\mathrm{PM}_{2.5}$ organic and total carbon (Fig. 13). Approximately 60\% and $70 \%$ of the days in July 2001 followed the OC and EC patterns, respectively, while around $60 \%$ of the days in January 2002 followed the OC and EC patterns. The features of the profiles varied seasonally, consistent with changes in meteorology.

Gas-phase species. Diurnal profiles for were also evident for several photochemically generated gas-phase species, including $\mathrm{O}_{3}, \mathrm{NO}$, and $\mathrm{NO}_{x}$ (Fig. 14). The features of these particular profiles also varied seasonally. Greater than $75 \%$ and $70 \%$ of the days followed 

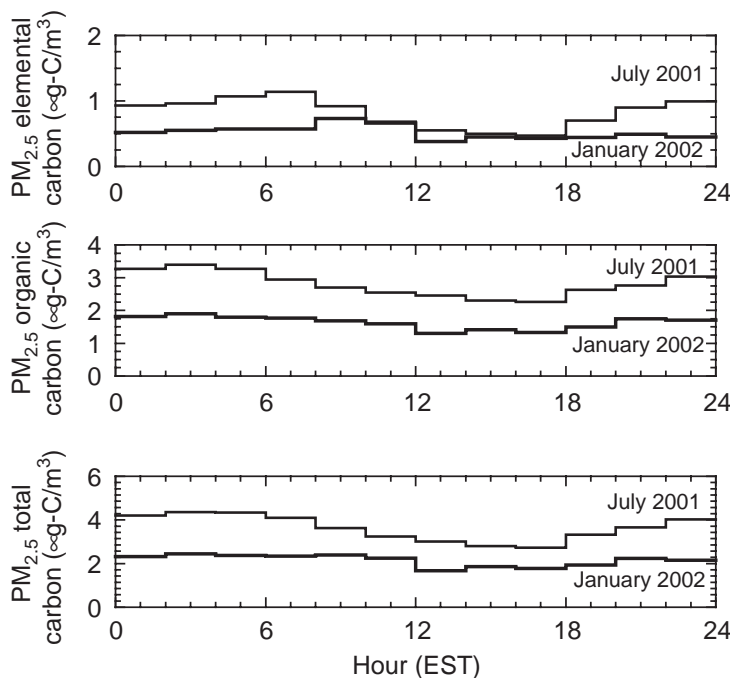

Fig. 13. Average diurnal variation in $\mathbf{P M}_{2.5}$ organic carbon, elemental carbon, and total carbon for July 2001 and January 2002 at the central site. Diurnal values were based on Sunset Labs in-situ carbon analyzer measurements.

these patterns in July 2001 and January 2002, respectively. The $\mathrm{CO}$ pattern is characterized by higher concentrations during the night, a peak during the morning rush hour and a lower concentration during the day. The pattern is more pronounced during the summer because of the stronger atmospheric mixing during the day.

\section{Conclusions}

PAQS is a comprehensive multidisciplinary set of projects in the Pittsburgh region, led by Carnegie Mellon University with contributions from academia, national laboratories, the private sector, and state and local air pollution agencies. The PAQS study design called for measurements to be made at a central site, at several satellite sites, and from two aircraft in order to characterize PM by examination of size, surface area, and volume distribution, chemical composition as a function of size and on a single particle basis, morphology, and temporal and spatial variability in the Pittsburgh region. Measurements made during the 14-month baseline study established the day-to-day character of the meteorology and ambient measurements, while measurements during the 2 intensive periods (ESP01 and ESP02) enhanced the temporal, spatial, and compositional detail of the baseline measurements. These measurements will allow the PAQS hypotheses to be systematically investigated.

Preliminary analyses of the overall dataset were presented in this paper. Nine days of relatively high
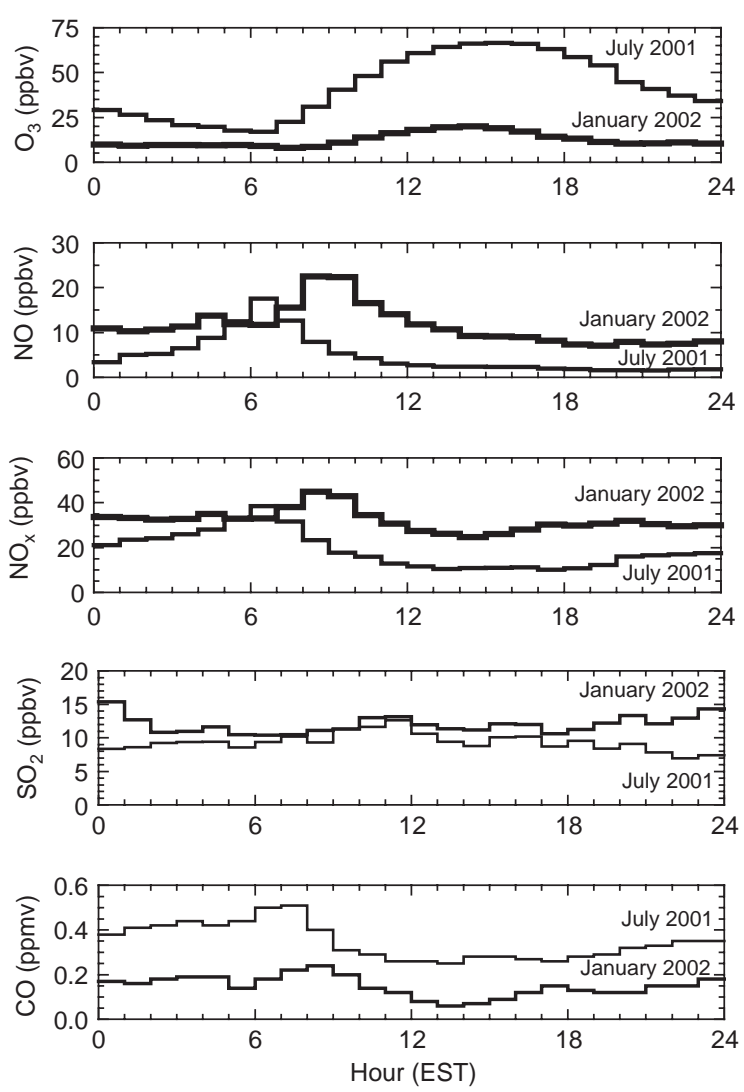

Fig. 14. Average diurnal variation in gas-phase concentrations of $\mathrm{O}_{3}, \mathrm{NO}, \mathrm{NO}_{x}, \mathrm{SO}_{2}$ and $\mathrm{CO}$ for July 2001 and January 2002 at the central site.

$\mathrm{PM}_{2.5}$ mass concentration experienced during PAQS at the central site were discussed, several of which formed multi-day episodes, and all of which occurred during the warmer summer months. Monthly average concentrations and diurnal profiles were also presented, to describe the features of the dataset, to emphasize the processes which affected the observed concentrations, and finally to demonstrate the consistency of the overall dataset. Changes in particular gaseous species or components of the $\mathrm{PM}_{2.5}$ mass were consistent with seasonal differences in average and diurnal patterns in meteorological measurements. On average, some of these patterns were not present or not as dramatic in January 2002 as they were in July 2001. Changes in the overall $\mathbf{P M}_{2.5}$ mass and light scattering were attributed to the major components of the mass, namely the sulfate component.

\section{Acknowledgements}

This research was conducted as part of the Pittsburgh Air Quality Study, which was supported by 
US Environmental Protection Agency under contract R82806101 and the US Department of Energy National Energy Technology Laboratory under contract DEFC26-01NT41017. This paper has not been subject to EPA's peer and policy review, and therefore does not necessarily reflect the views of the Agency. No official endorsement should be inferred.

\section{References}

Blando, J.D., Porcja, R.J., Turpin, B.J., 2001. Issues in the quantitation of functional groups by FTIR spectroscopic analysis of impactor-collected aerosol samples. Aerosol Science and Technology 35, 899-908.

Cabada, J.C., Khlystov, A., Wittig, A.E., Pandis, S.N., 2004a. Fine particle light scattering reconstruction and measurements at PAQS. Journal of Geophysical Research-Atmospheres, submitted for publication.

Cabada, J.C., Pandis, S.N., Subramanian, R., Robinson, A.L., Polidori, A., Turpin, B., 2004b. Estimating the secondary organic aerosol contribution to $\mathrm{PM}_{2.5}$ using the EC tracer method. Aerosol Science and Technology, in press.

Cabada, J.C., Takahama, S., Khlystov, A., Pandis, S.N., Rees, S., Davidson, C.I., Robinson, A.L., 2004c. Mass size distributions and size resolved chemical composition of fine particulate matter at the Pittsburgh Supersite. Atmospheric Environment, this issue, doi: 10.1016/j.atmosenv. 2004.03.004.

Carson, P.G., Johnston, M.V., Wexler, A.S., 1997. Laser desorption ionization of ultra fine aerosol particles. Rapid Communications of Mass Spectroscopy 11, 993-996.

Code of Federal Regulations, 2002. Title 40, Subchapter C Part 50.

Demoz, B., Collett Jr., J.L., Daube Jr., B.C., 1996. On the Caltech active strand cloudwater collectors. Atmospheric Research 41, 47-62.

Eatough, D.J., Eatough, N.L., Obeidi, F., Pang, Y., Modey, W., Long, R., 2001. Continuous determination of $\mathrm{PM}_{2.5}$ mass, including semi-volatile species. Aerosol Science and Technology 34, 1-8.

Erisman, J.W., Otjes, R., Hensen, A., et al., 2001. Instrument development and application in studies and monitoring of ambient ammonia. Atmospheric Environment 35, 1913-1922.

Ge, Z., Wexler, A.S., Johnston, M.V., 1998. Laser desorption/ ionization of single ultra fine multicomponent aerosols. Environmental Science and Technology 32, 3218-3223.

Harris, J.M., Kahl, J.D.W., 1994. An analysis of 10-day isentropic flow patterns for Barrow, Alaska: 1985-1992. Journal of Geophysical Research 99, 25845-25955.

Jaffrezo, J.L., Calas, T., Bouchet, M., 1998. Carboxylic acids measurements with ionic chromatography. Atmospheric Environment 32, 2705-2708.

Jayne, J.T., Leard, D.C., Zhang, X., Davidovits, P., Smith, K.A., Kolb, C.E., Worsnop, D.R., 2000. Development of an aerosol mass spectrometer for size and composition analysis of submicron particles. Aerosol Science and Technology 33, 49-70.

Jimenez, J.L., Jayne, J.T., Shi, Q., Kolb, C.E., Worsnop, D.R., Yourshaw, I., Seinfeld, J.H., Flagan, R.C., Zhang, X.,
Smith, K.A., Morris, J., Davidovits, P., 2003. Ambient aerosol sampling with an aerosol mass spectrometer. Journal of Geophysical Research-Atmospheres (108), ACE 13-1-13-22.

Khlystov, A., Wyers, G.P., Slanina, J., 1995. The steam-jet aerosol collector. Atmospheric Environment 29, 2229-2234.

Khlystov, A., Wittig, A.E., Davidson, C., 2001. Quality assurance project plan for the Pittsburgh air quality study. Report prepared for the U.S.E.P.A., Research Triangle Park, NC.

Khlystov, A., Stanier, C., Pandis, S.N., 2003. An algorithm for combining electrical mobility and aerodynamic size distributions data when measuring ambient aerosol. Aerosol Science and Technology, submitted for publication.

Kidwell, C.B., Ondov, J.M., 2001. Development and evaluation of a prototype system for collecting sub-hourly ambient aerosol for chemical analysis. Aerosol Science and Technology 35, 596-601.

Lazrus, A.L., Kok, G.L., Lind, J.A., Gitlin, S.N., Heikes, B.G., Shetter, R.E., 1986. Automated fluorometric method for hydrogen peroxide in air. Analytical Chemistry 58, 594-597.

Lewis, C.W., Stevens, R.K., Rasmussen, R.A., Caldelino, C.A., Pierce, T.E., 1999. Biogenic fraction of ambient VOC: comparison of radiocarbon, chromatographic, and emission inventory estimates for Atlanta, Georgia. Journal of the Air and Waste Management Association 49, 299-307.

Lipsky, E., Stanier, C.O., Pandis, S.N., Robinson, A.L., 2002. Effects of sampling conditions on the size distribution of fine particulate matter emitted from a pilot-scale pulverizedcoal combustor. Energy and Fuels 16, 302-310.

Malm, W.C., Gebhard, K.A., Molenar, J., Cahill, T., Eldred, R., Huffman, D., 1994a. Examining the relationship between atmospheric aerosols and light extinction at Mount Rainier and North Cascades National Parks. Atmospheric Environment 28, 247-260.

Malm, W.C., Sisler, J.F., Huffman, D., Eldred, R.A., Cahill, T.A., 1994b. Spatial and seasonal trends in particle concentration and optical extinction in the United States. Journal of Geophysical Research 99, 1347-1370.

MSP Corp., Inc., 1998. Operator's Manual for the MicroOrifice Uniform Deposit Impactor. Shoreview, MN.

NIOSH, 1996. Elemental carbon (diesel exhaust). In: NIOSH Manual of Analytical Methods. National Institute of Occupational Safety and Health, Cincinnati, OH.

Slanina, J., Ten Brink, H.M., Otjes, R.P., Even, A., Jongejan, P., Khlystov, A., Waijers-Ijpelaan, A., Hu, M., 2001. The continuous analysis of nitrate and ammonium in aerosols by the Steam Jet Aerosol Collector (SJAC): extension and validation of the methodology. Atmospheric Environment 35, 2319-2330.

Stanier, C.O., Khlystov, A., Chan, R., Mandiro, M., Pandis, S.N., 2004a. A method for the in-situ measurement of fine aerosol water content of ambient aerosols: the Dry-Ambient Aerosol Size Spectrometer (DAASS). Aerosol Science and Technology, in press.

Stanier, C.O., Khlystov, A., Pandis, S.N., 2004b. Nucleation events during the Pittsburgh air quality study: description and relation to key meteorological, gas-phase, and aerosol parameters. Aerosol Science and Technology, in press.

Stolzenburg, M.R., Hering, S.V., 2000. A new method for the automated measurement of atmospheric fine 
particle nitrate. Environmental Science and Technology 34, 907-914.

Subramanian, R., Khlystov, A.Y., Cabada, J.C., Robinson, A.L., 2003. Positive and negative artifacts in particulate organic carbon measurements with denuded and undenuded sampler configurations. Aerosol Science and Technology, submitted for publication.

Takahama, S., Khlystov, A., Wittig, A.E., Davidson, C., Pandis, S.N., 2004. Partitioning of nitrate during the Pittsburgh air quality study and implications of timeaveraging. Journal of Geophysical Research-Atmospheres, submitted for publication.

Tang, W., Raymond, T., Wittig, B., Davidson, C., Pandis, S.N., Robinson, A., Crist, K., 2004. Spatial variations of $\mathrm{PM}_{2.5}$ during the Pittsburgh air quality study. Aerosol Science and Technology, in press.

Thermo Andersen, 1999. Operator's Manual for $\mathrm{PM}_{10}$ High Volume Sampler. Smyrna, GA.

Tolocka, M.P., Solomon, P.A., Mitchell, W., Norris, G.A., Gemmill, D.B., Wiener, R.W., Vanderpool, R.W., Homolya, J.B., Rice, J., 2001. East versus West in the US: chemical characteristics of $\mathrm{PM}_{2.5}$ during the winter of 1999. Aerosol Science and Technology 34, 88-96.

Turpin, B.J., Cary, R.A., Huntzicker, J.J., 1990. An in-situ time-resolved analyzer for aerosol organic and elemental carbon. Aerosol Science and Technology 12, 161-171.

U.S.E.P.A., 1991. EPA Method 200.8 (rev 4.4): Determination of trace elements in waters and wastes by Inductively
Coupled Plasma Mass Spectrometry. Research Triangle Park, NC.

U.S.E.P.A., 1994. EPA Method 6020: Inductively Coupled Plasma Mass Spectrometry. Research Triangle Park, NC.

U.S.E.P.A., 1998. EPA Quality assurance guidance 2.12: Monitoring $\mathrm{PM}_{2.5}$ in ambient air using designated references or Class I equivalence methods. Research Triangle Park, NC.

U.S.E.P.A., 1999. EPA Method 625 (rev R-96/01a): Compendium of methods for the determination of inorganic compounds in ambient air. Research Triangle Park, NC.

Willeke, K., Baron, P.A., 1993. Aerosol Measurement Principles, Techniques, and Applications. Van Nostrand Reinhold, New York.

Wittig, A.E., Davidson, C., 2003. Quality assurance final report for the Pittsburgh Air Quality Study (PAQS) supersite. Report prepared for the U.S.E.P.A., Research Triangle Park, NC.

Wittig, A.E., Takahama, S., Khlystov, A., Pandis, S.N., Hering, S., Kirby, B., Davidson, C., 2003. Semi-continuous $\mathrm{PM}_{2.5}$ inorganic composition measurements during the Pittsburgh Air Quality Study. Atmospheric Environment, submitted for publication.

Zhang, J., Chameides, W.L., Weber, R., Cass, G., Orsini, D., Edgerton, E., Jongejan, P., Slanina, J., 2002. An evaluation of the thermodynamic equilibrium assumption for fine particulate composition: nitrate and ammonium during the 1999 Atlanta supersite experiment. Journal of Geophysicals Research-Atmospheres (108), SOS 2-1-2-11. 